\title{
A new Readout Control System for the LHCb Upgrade at CERN
}

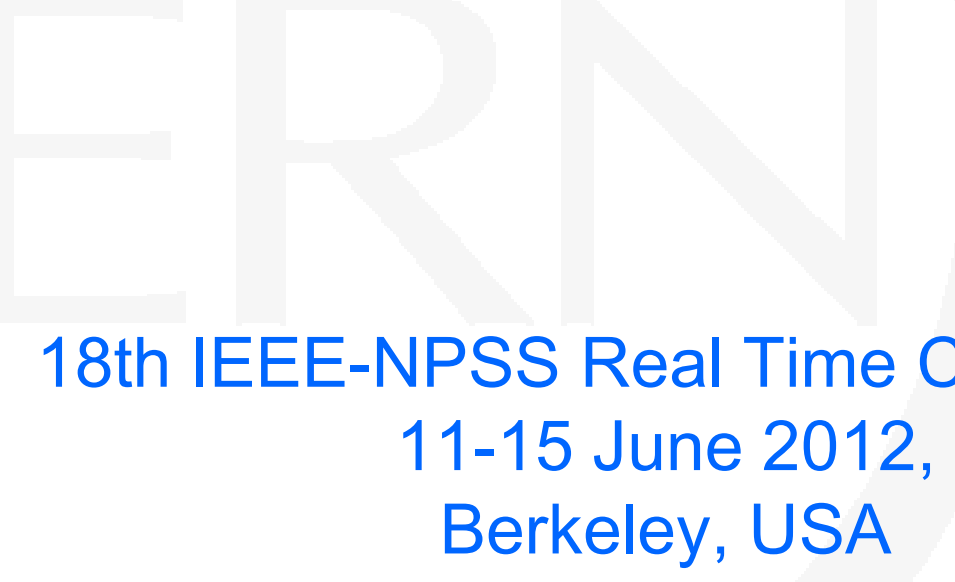

Federico Alessio, CERN Richard Jacobsson, CERN 


\section{The upgrade of the $\mathrm{LHCb}$ experiment}

ONLINE

\section{LHCb in 2012}

$\checkmark$ Instantaneous luminosity in IP of $4 \times 10^{32} \mathrm{~cm}^{-2} \mathrm{~s}^{-1}$ (factor 50 less than nominal LHC lumi)

$\checkmark \quad$ Expected $\int \mathscr{L}=5-10 \mathrm{fb}^{-1}$ collected after 5 years of operations

$\rightarrow$ Probe/measure NewPhysics at $10 \%$ level of sensitivity

$\rightarrow$ Measurements limited by statistics and detector itself

$\rightarrow$ World best measurements in flavor physics and rare decays already performed in 2011-2012

Upgrade! (During LHC Long Shutdown 1 in 2018)

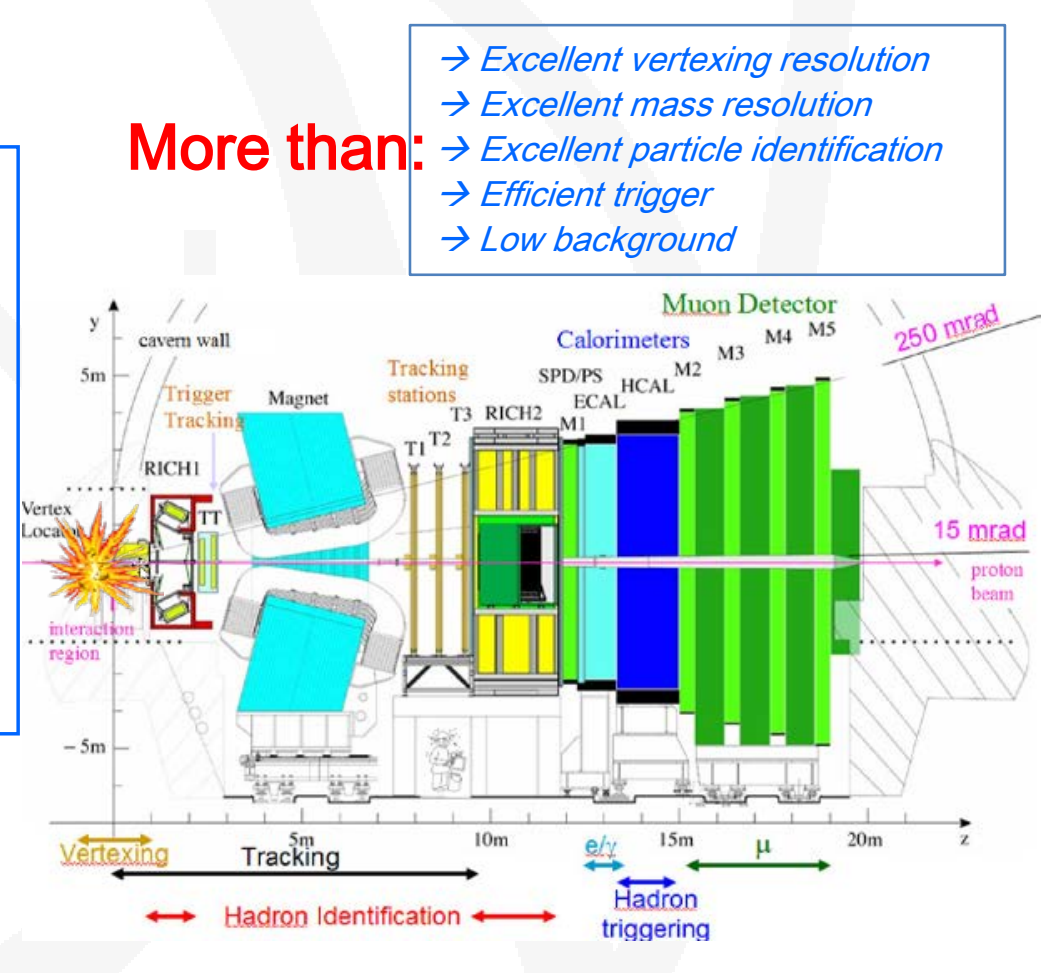

\section{S-LHCb in 2018-19}

$\checkmark$ Collect $\int \mathscr{L}=50 \mathrm{fb}^{-1} \rightarrow$ a factor 10 increase in data sample and in reasonable time

$\rightarrow$ probe NewPhysics down to a percent level

$\checkmark$ Increase luminosity by a factor $10 @ \mathrm{LHCb}$, up to $2 \times 10^{33} \mathrm{~cm}^{-2} \mathrm{~s}^{-1}$

$\rightarrow 28 \mathrm{MHz}$ S-LHCb effective collisions rate vs. $1 \mathrm{MHz} \mathrm{LHCb}$

$\rightarrow 1 \mathrm{MHz}$ bb-pair rate @ S-LHCb vs. $100 \mathrm{KHz} @$ LHCb 


\section{LHCb The challenges of the LHCb upgrade}

ONLINE

$\checkmark \quad$ Pile-up (N): number of interactions per LHC bunch-bunch crossing

$\rightarrow$ LHCb designed for $<$ Nmax $>=1$

$\rightarrow<\mathrm{N}>=1 @ 2 \times 10^{32} \mathrm{~cm}^{-2} \mathrm{~s}^{-1} /<\mathrm{N}>=4 @ 20 \times 10^{32} \mathrm{~cm}^{-2} \mathrm{~s}^{-1}$

$\checkmark$ Higher radiation damages over time

$\checkmark$ Spill-over not minimized

$\checkmark$ Current first-level trigger limited for hadronic modes at $>2 \times 10^{32} \mathrm{~cm}^{-2} \mathrm{~s}^{-1}$

$\rightarrow 25 \%$ efficiency vs. $75 \%$ for muonic modes

$\checkmark \quad$ Full readout of $28 \mathrm{MHz}$ of bunch-bunch crossing

$\rightarrow$ current first level trigger selects only $1 \mathrm{MHz}$ of events

\section{Upgrade!}

$\checkmark$ New technologies for sub-detectors to be replaced

$\rightarrow$ More radiation hard, Reduced spill-over, Improved granularity

$\checkmark$ Continuous $40 \mathrm{MHz}$ Trigger-free Readout Architecture

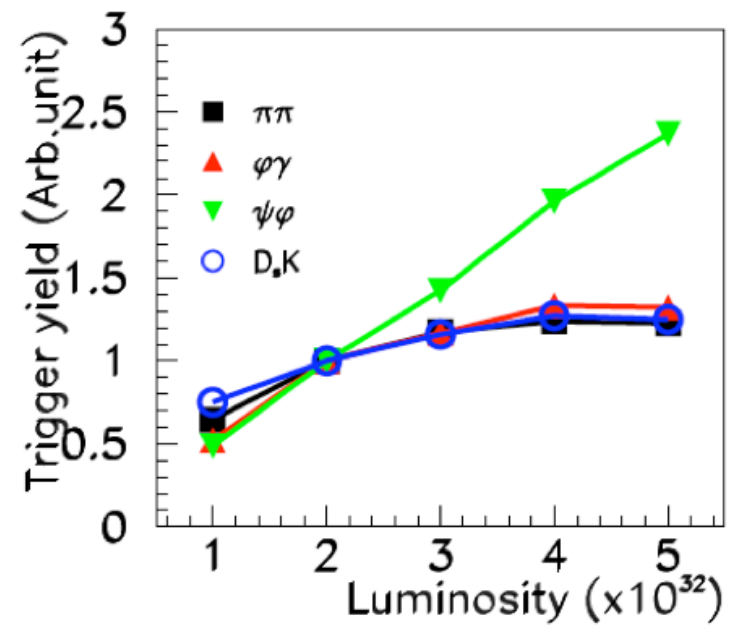

$\rightarrow$ all detector data passed through the readout network

$\rightarrow$ fully software trigger analyzing events at $40 \mathrm{MHz}$ 


\section{KHCb Upgraded LHCb Readout System}

ONLINE

First-level triggered events

Detector

25ns FE

$1 \mathrm{MHz} \rightarrow 28 \mathrm{MHz}$

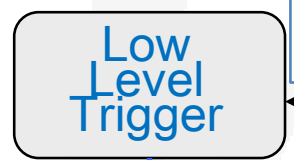

\begin{tabular}{|l|l|l|l|l|l|}
\hline VELO ST OT RICH ECal HCal Muon \\
\hline
\end{tabular}

Use of bidirectional links

to/from FE

Based on CERN GigaBit

Transceiver (GBT)

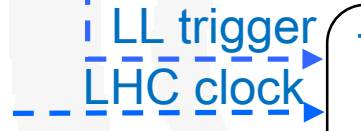

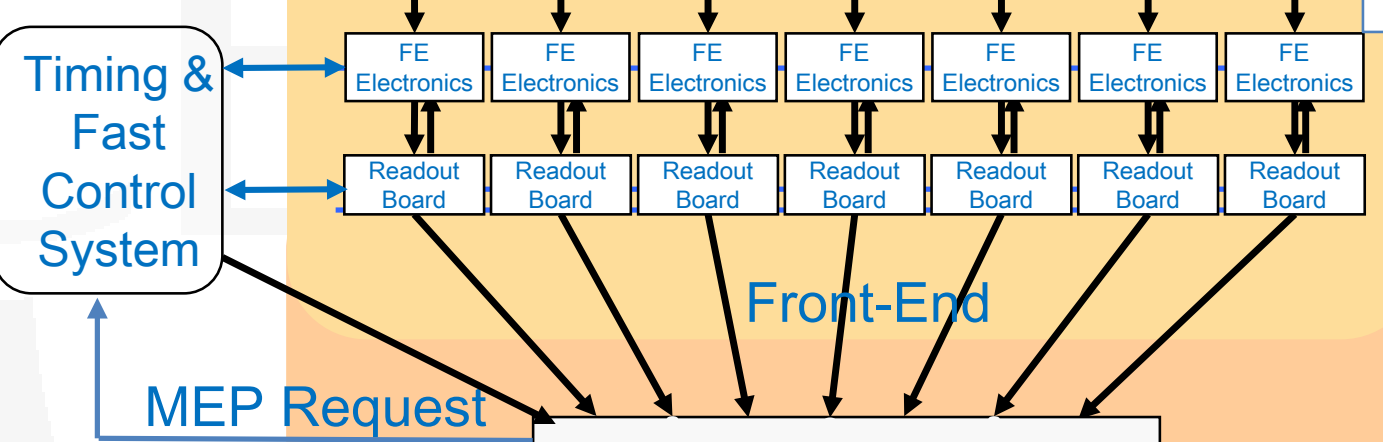
$\sim 400$ Readout
Boards
(TELL40)

Output rate of processed events $20 \mathrm{kHz}$

(currently is $4.5 \mathrm{kHz}$ )

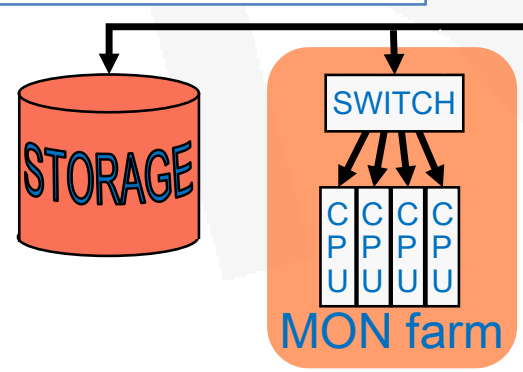

READOUT NETWORK

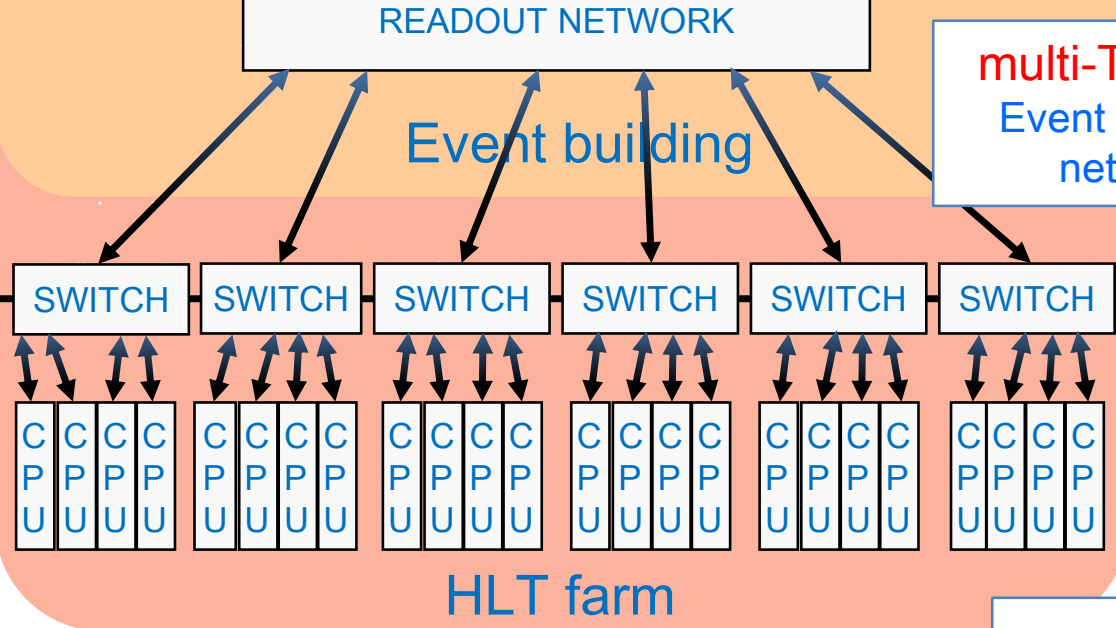

$$
>5000 \mathrm{CPU}
$$

processing multi-core nodes

O(50k cores) 


\section{LHCb \\ Upgraded LHCb Readout System}

Iricp

ONLINE

Fully trigger-free $40 \mathrm{MHz}$ readout architecture!

_. 느븓 c clock

Output rate of processed events $20 \mathrm{kHz}$

(currently is $4.5 \mathrm{kHz}$ )

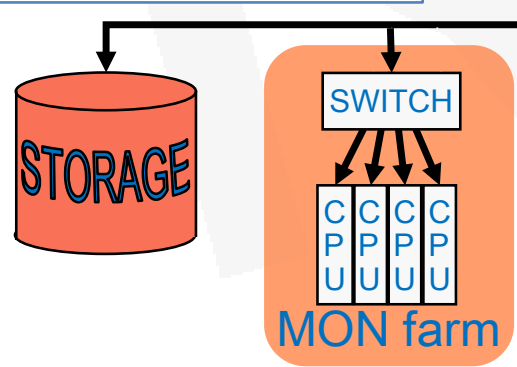

\section{5ns FE \\ Detector}

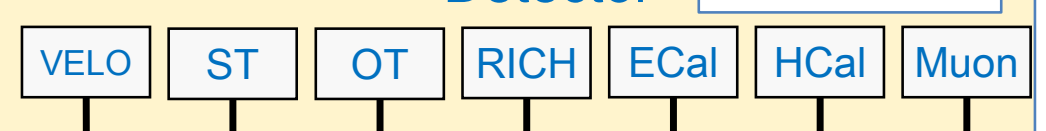

Use of bidirectional links to/from FE

Based on CERN GigaBit Transceiver (GBT)

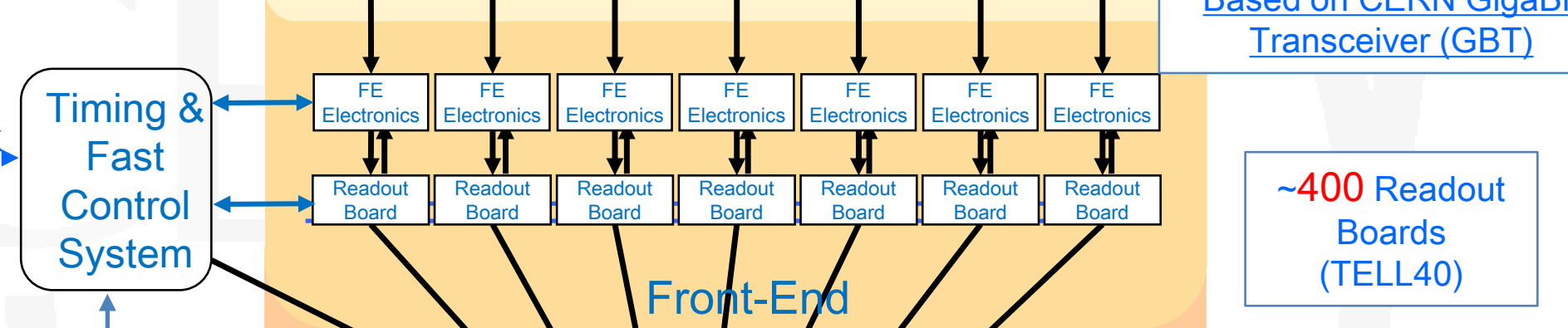

READOUT NETWORK

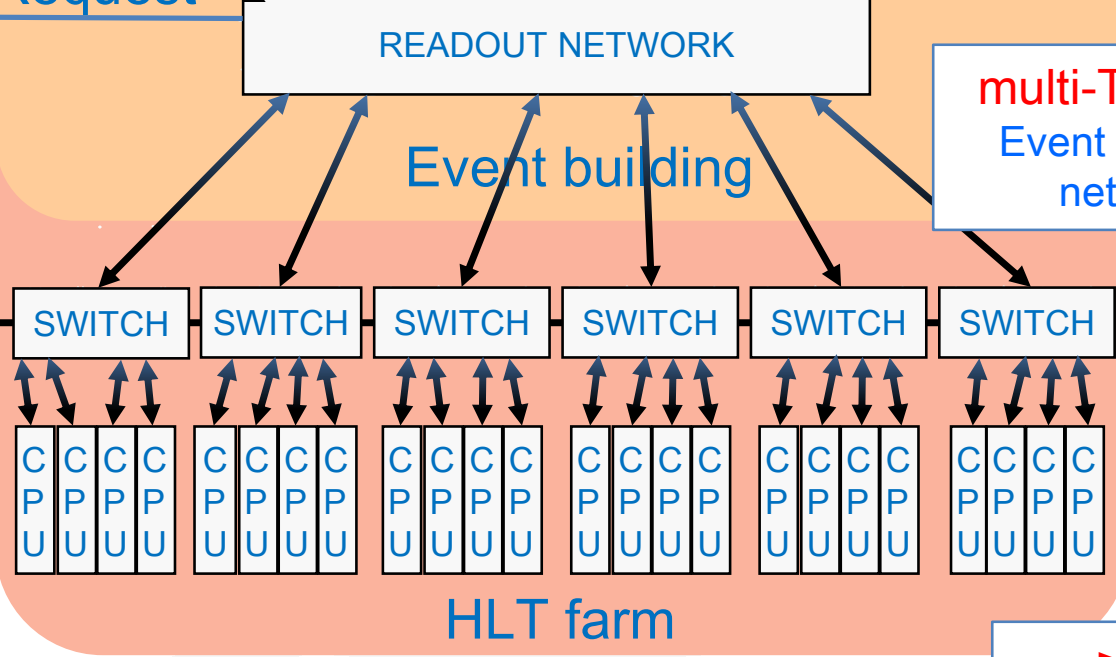




\section{LHCh \\ Upgraded LHCb Readout System}

ONLINE

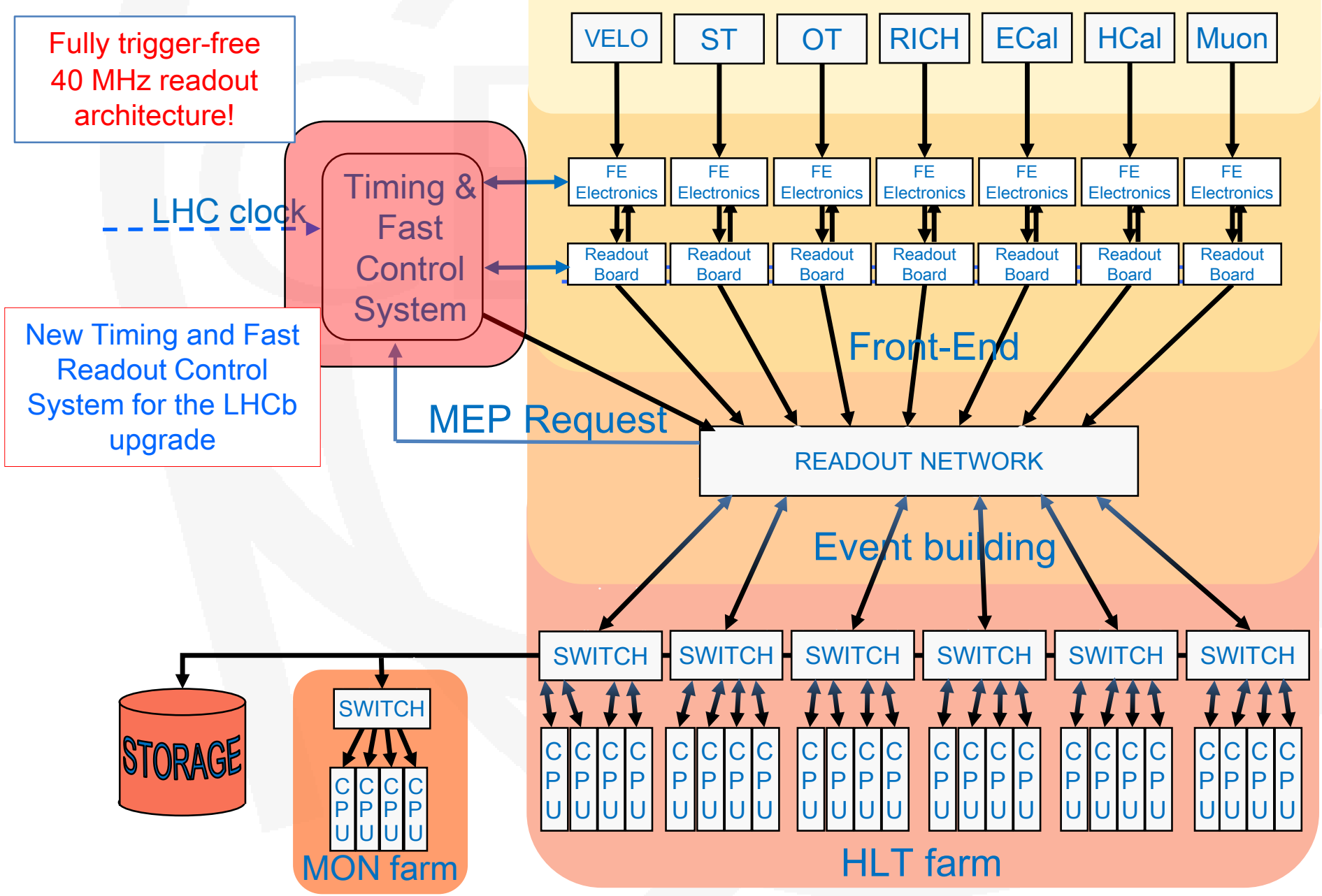




\section{System and functional requirements of the new Readout Control system}

1. Bidirectional communication network

2. Clock jitter, and phase and latency control

$\checkmark \quad$ At the FE, but also at the Readout Boards and between S-TFC boards

3. LHC interfaces

4. Low-Level-Trigger input

5. Events rate control

6. Destination control for the event packets

7. Event data bank

$\checkmark$ Infomation about transmitted events

8. Sub-detectors calibration triggers

9. Partitioning to allow running with any ensemble and parallel partitions 10. Support for old Timing and Trigger based distribution system 11. Test-bench support + flexibility as it should be ready for testing!

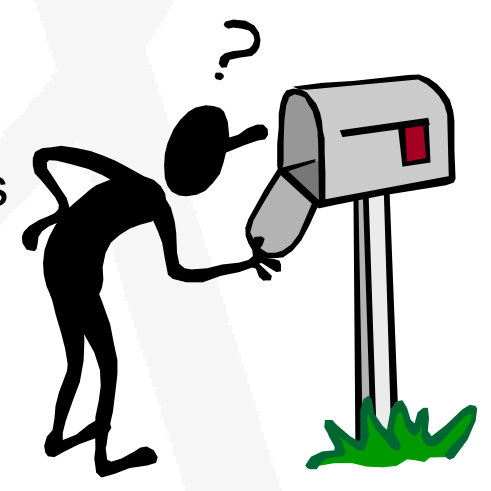




\section{LHCb LOWLEVEL A new LHCb Readout Control System}

TRIGGER LHC Interfaces

ONLINE

TRIGGER

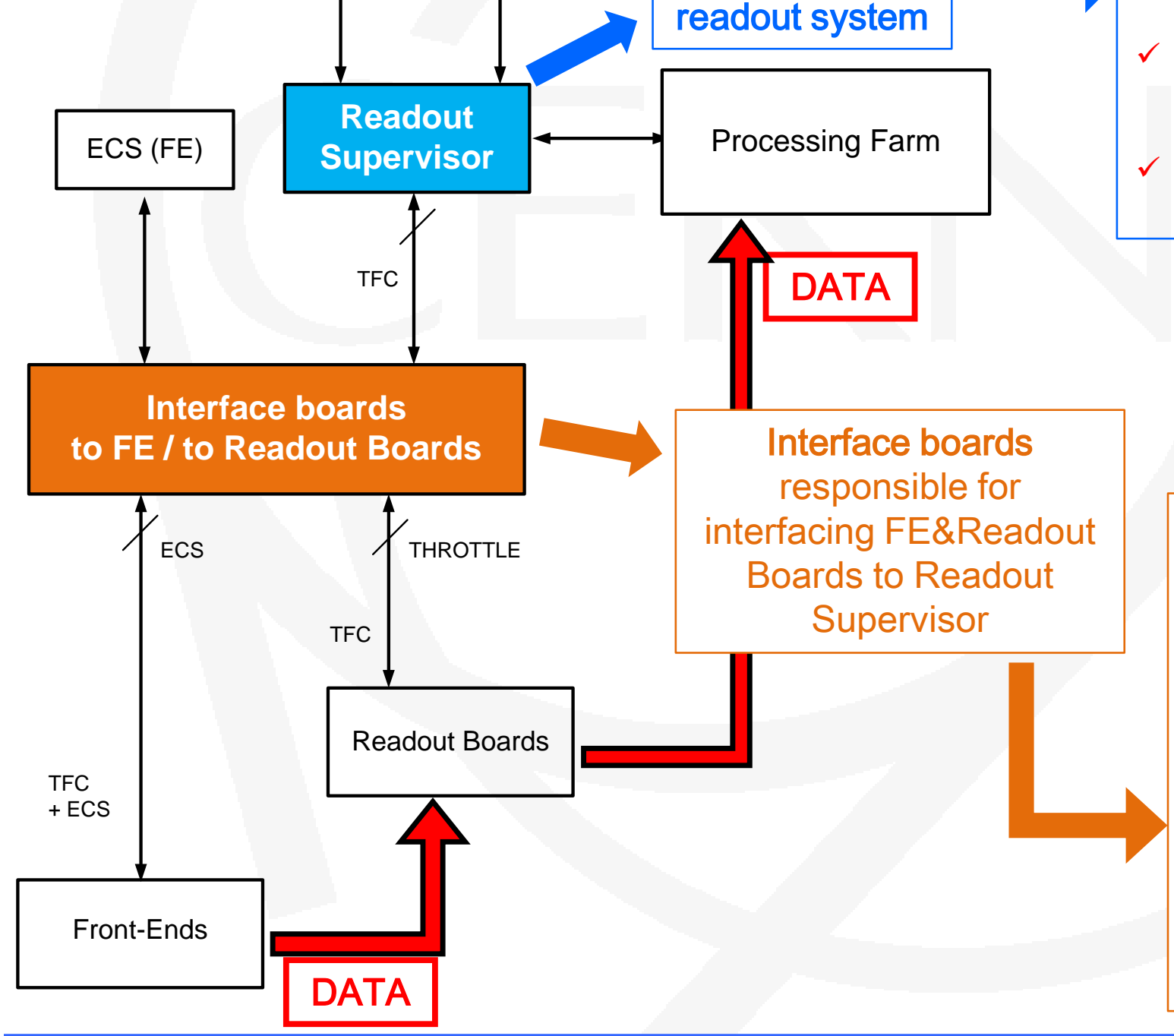

18th IEEE NPSS Real Time Conference,

11-15 June 2012, Berkeley, USA $\checkmark$ Distributes timing, trigger and synchronous commands

Manages the dispatching of events to the Processing Farm

Rate regulates the system taking into account back-pressure/throttle $\checkmark \quad$ Fan-out timing and control information (TFC) to Readout Boards

$\checkmark \quad$ Fan-in Throttle information from Readout Boards

$\checkmark \quad$ Distributes TFC information to FE

$\checkmark \quad$ Distributes control configuration data to FE

$\checkmark \quad$ Receives control monitoring data from FE 


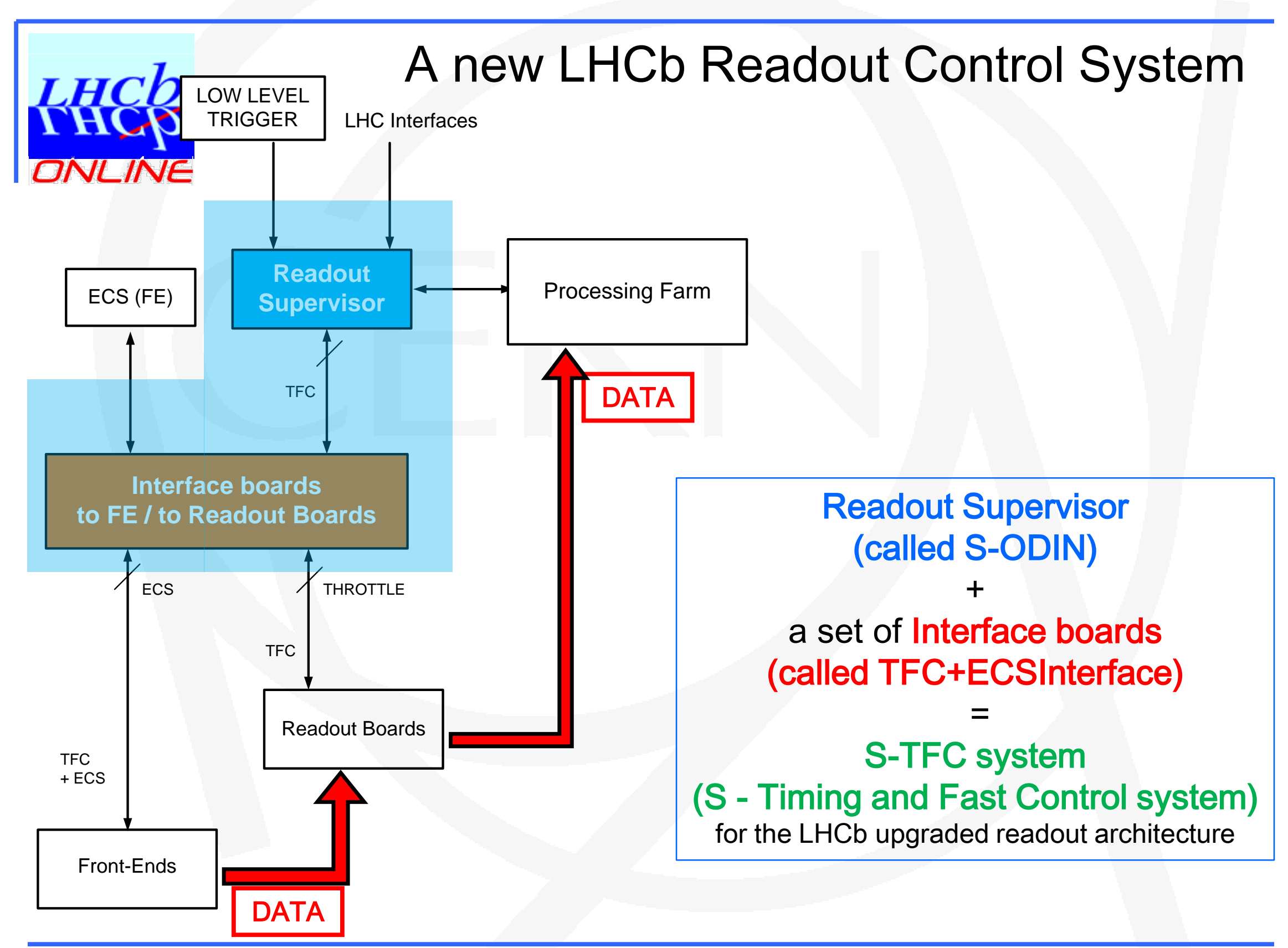




\section{The physical S-TFC system}

ONLINE

LHC clock, LHC interfaces

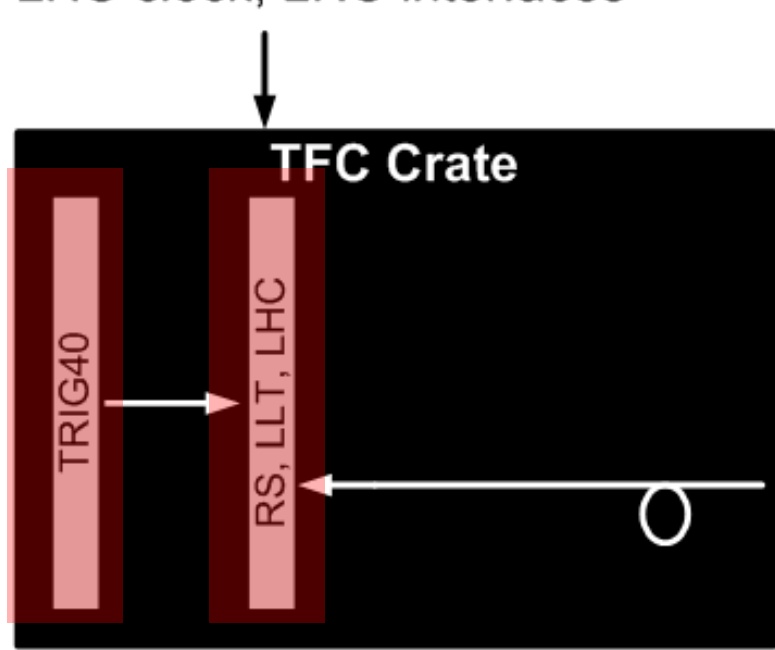

RS $\rightarrow$ common AMC card LLT $\rightarrow$ common AMC card Readout board $\rightarrow$ common AMC card LHC Interfaces $\rightarrow$ common AMC card with special mezzanine

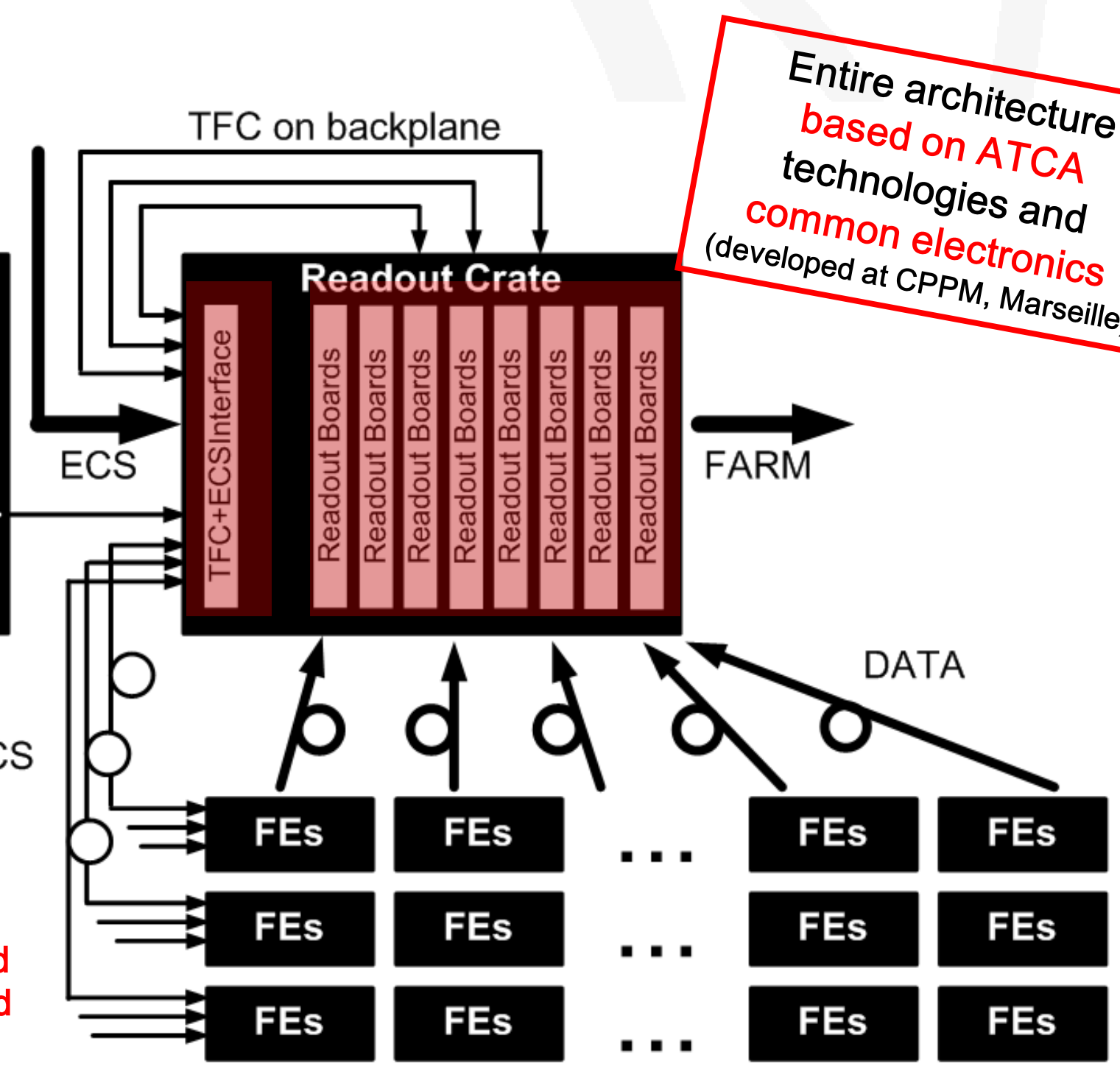




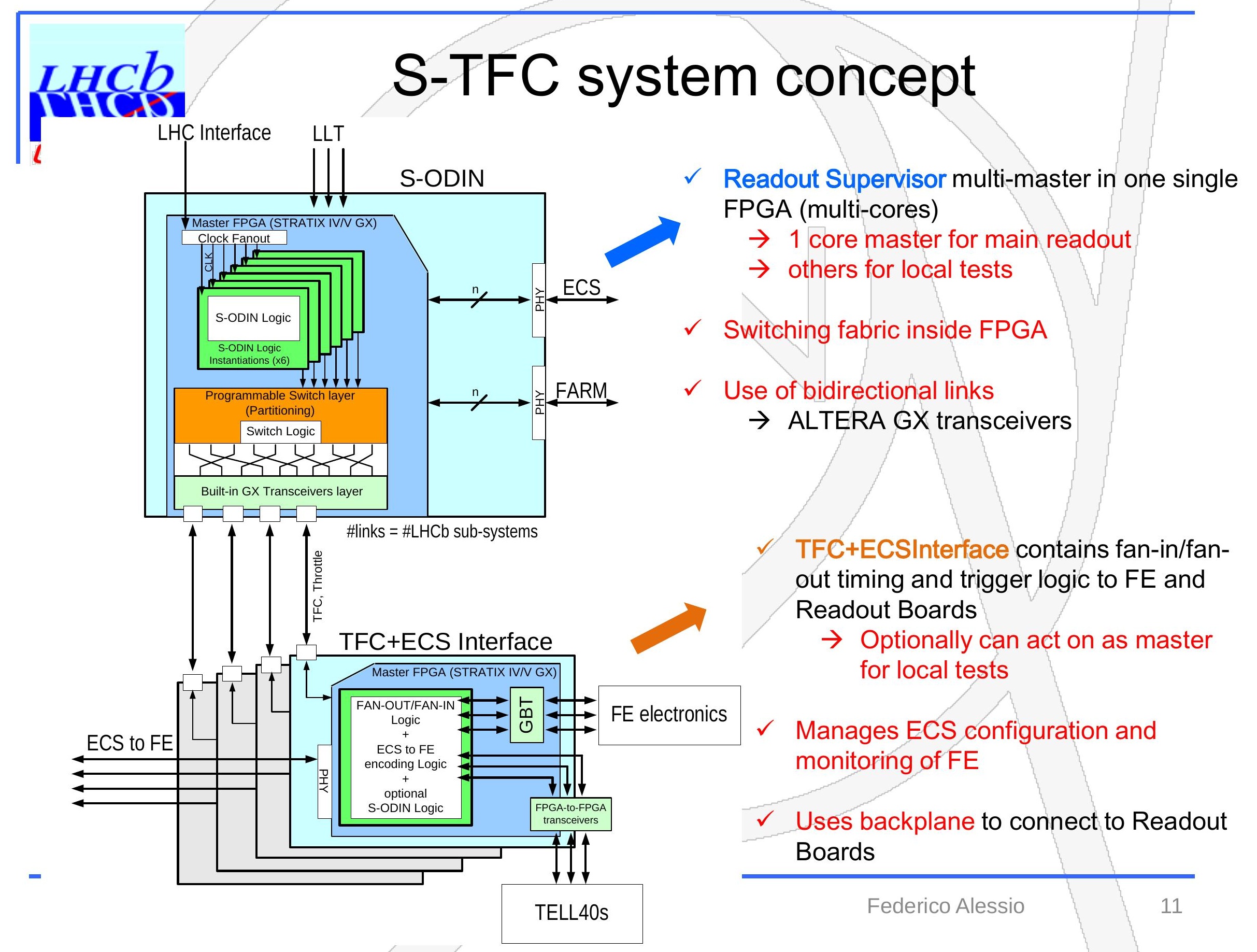




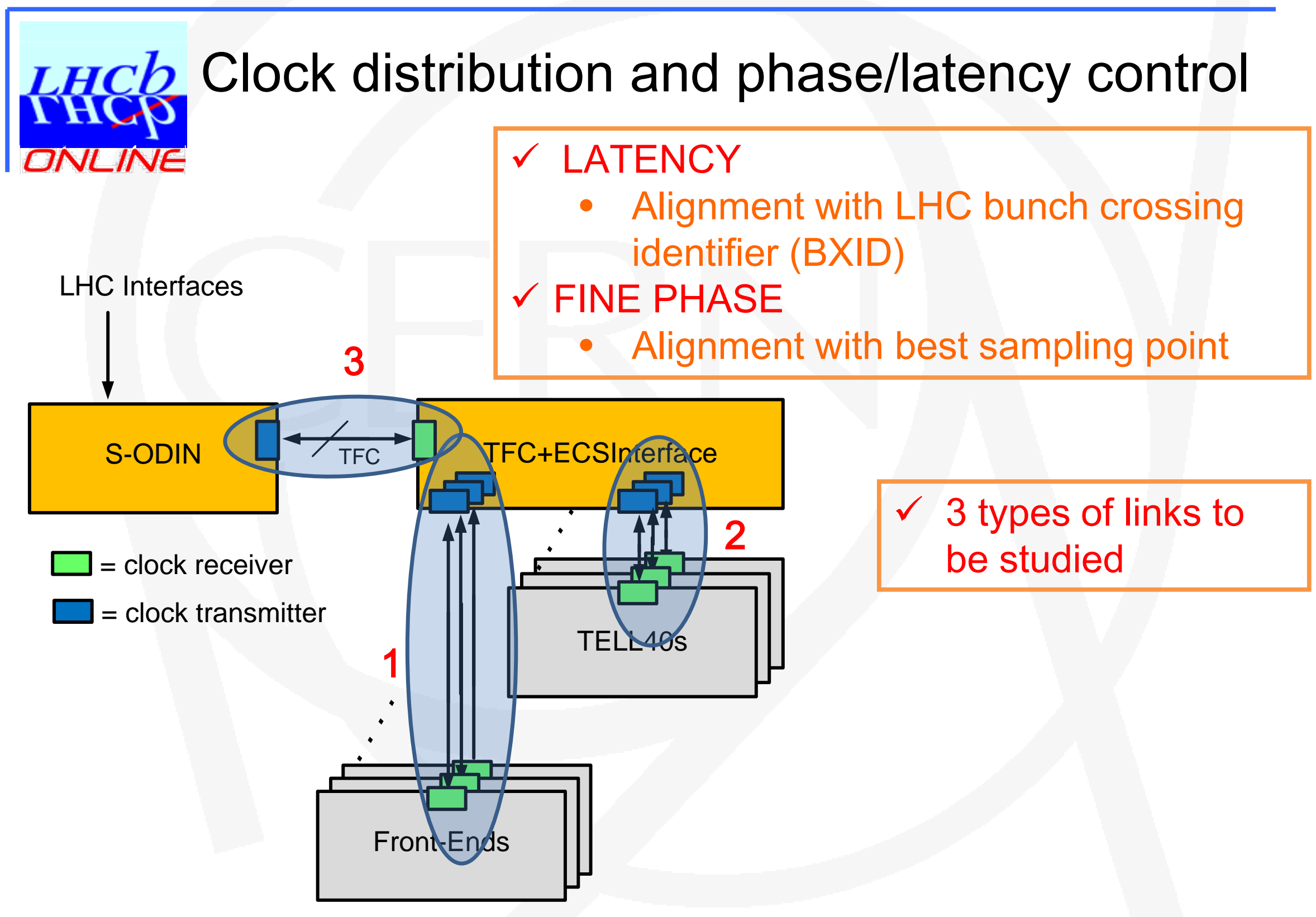




\section{LHCb Clock distribution and phase/latency control \\ THCP}

\section{ONLINE}

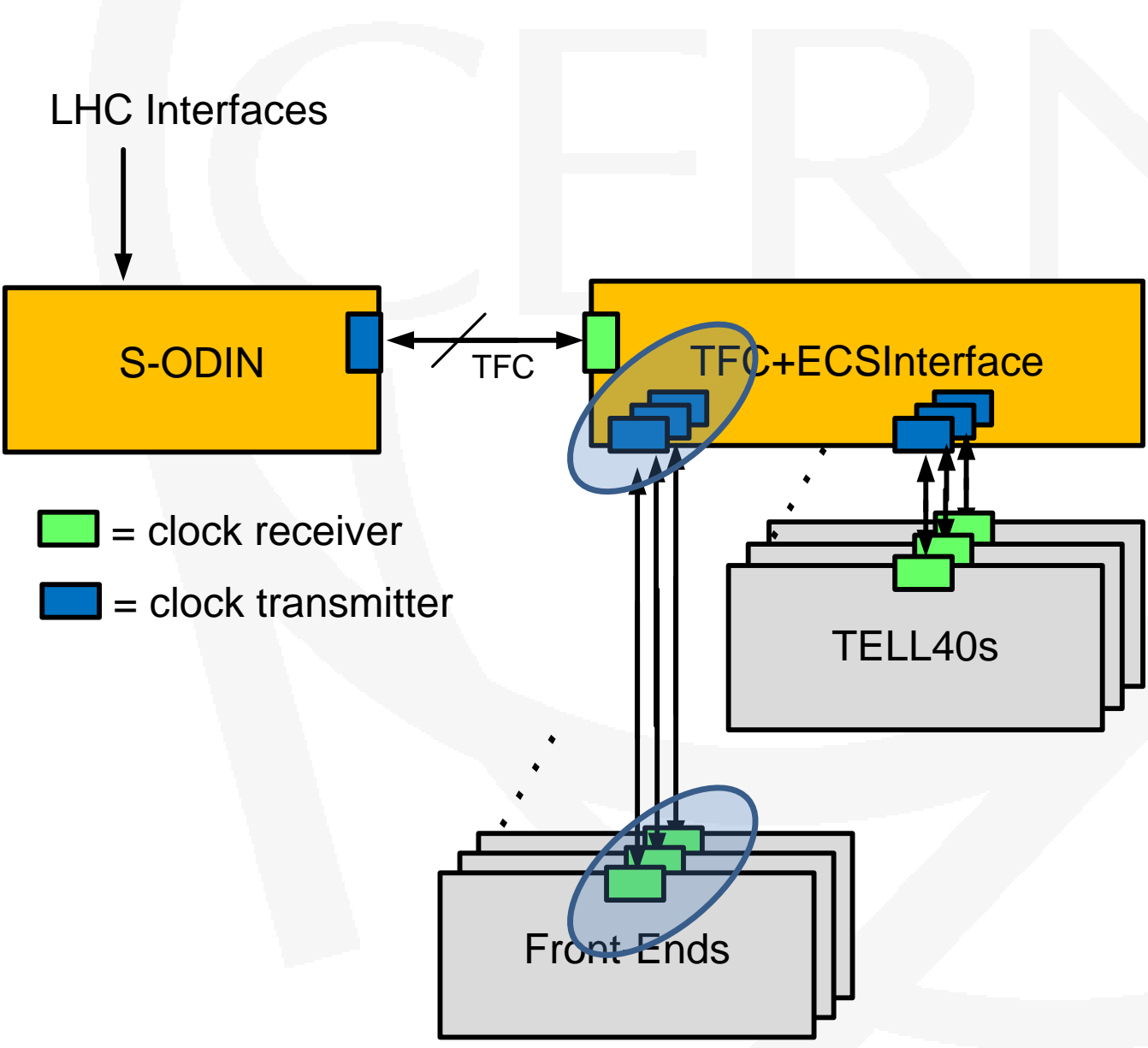

1. at the FE: CERN GBT $\rightarrow$ Does the job for us

$\rightarrow$ control of fine phase + latency at the FE + minimize jitter

$\rightarrow$ No problem in ECS monitoring

- Simply decoding GBT protocol in TFC+ECSInterface FPGA $\rightarrow$ No need of fine phase or latency control for ECS. 


\section{LHCb Clock distribution and phase/latency control}

\section{ONLINE}

LHC Interfaces
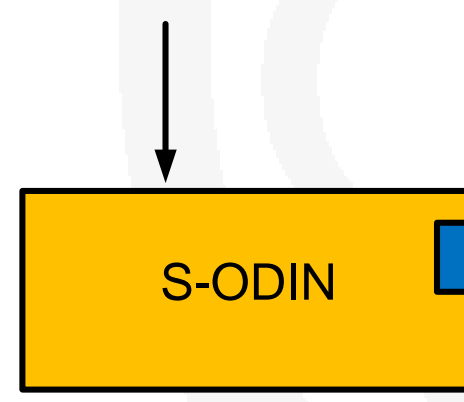

$\square$ = clock receiver

$\square=$ clock transmitter
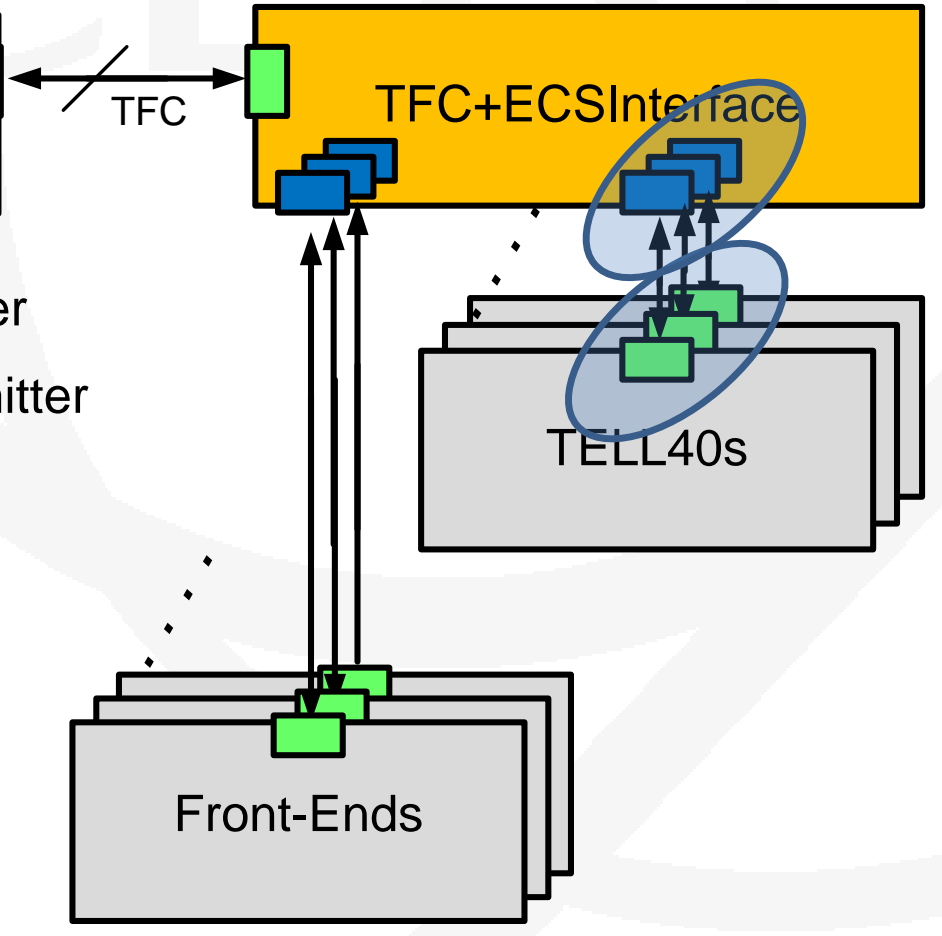

2. ATCA backplane

$\rightarrow$ Does the job for us

$\rightarrow$ control of latency

$\rightarrow$ jitter and fine phase less of an issue

$\rightarrow$ Effectively is an FPGA-to-FPGA link on backplane dedicated lines

$\rightarrow$ To be tested; jitter on backplane! 


\section{$\mathrm{LHCl}$ Clock distribution and phase/latency control \\ IONLINE}

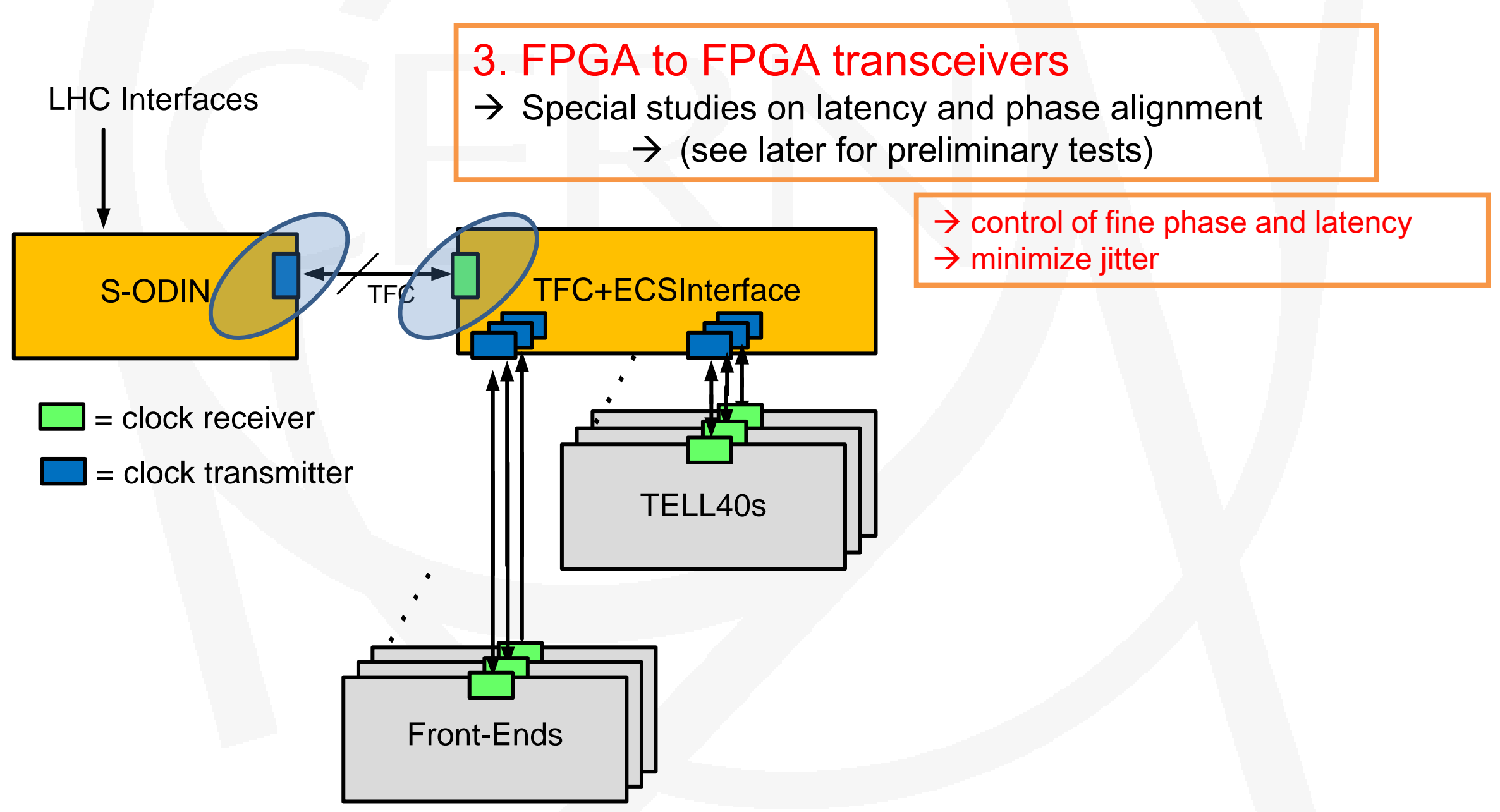




\section{Validation tests}

First preliminary tests on phase/latency control using:

1. First AMC prototype with ALTERA Stratix IV

2. First Stratix IV low level interfaces

$\rightarrow$ Nios + board resources interfaces

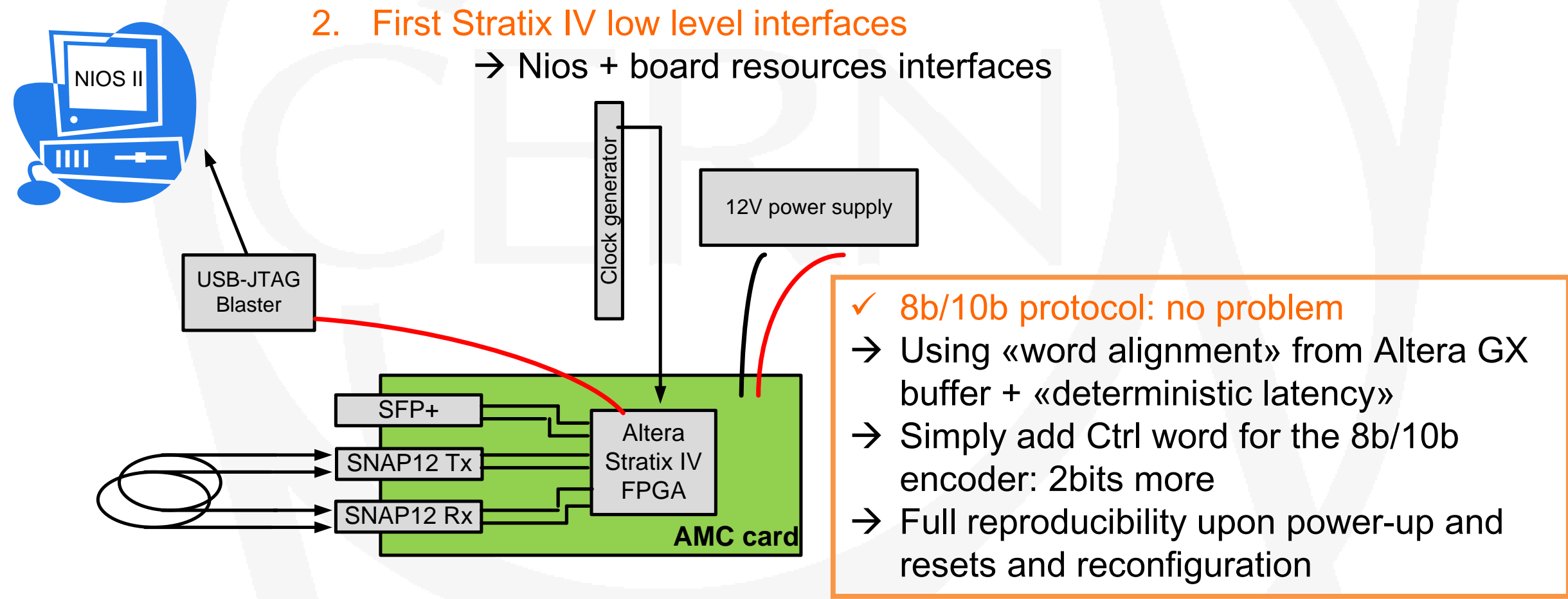

$\checkmark$ FPGA-to-FPGA GBT protocol: ok, but needs special frame alignment

$\rightarrow$ No deterministic latency if no special words are sent!

$\rightarrow$ Needs a special word (10 bits minimum) at power-up/after reset/after reconfiguration for the GX buffer to realign to the beginning of the frame + "deterministic latency»

- First preliminary tests were ok, but needs more validation 
Relay/merge block logic:

TELL40s (44 bits)

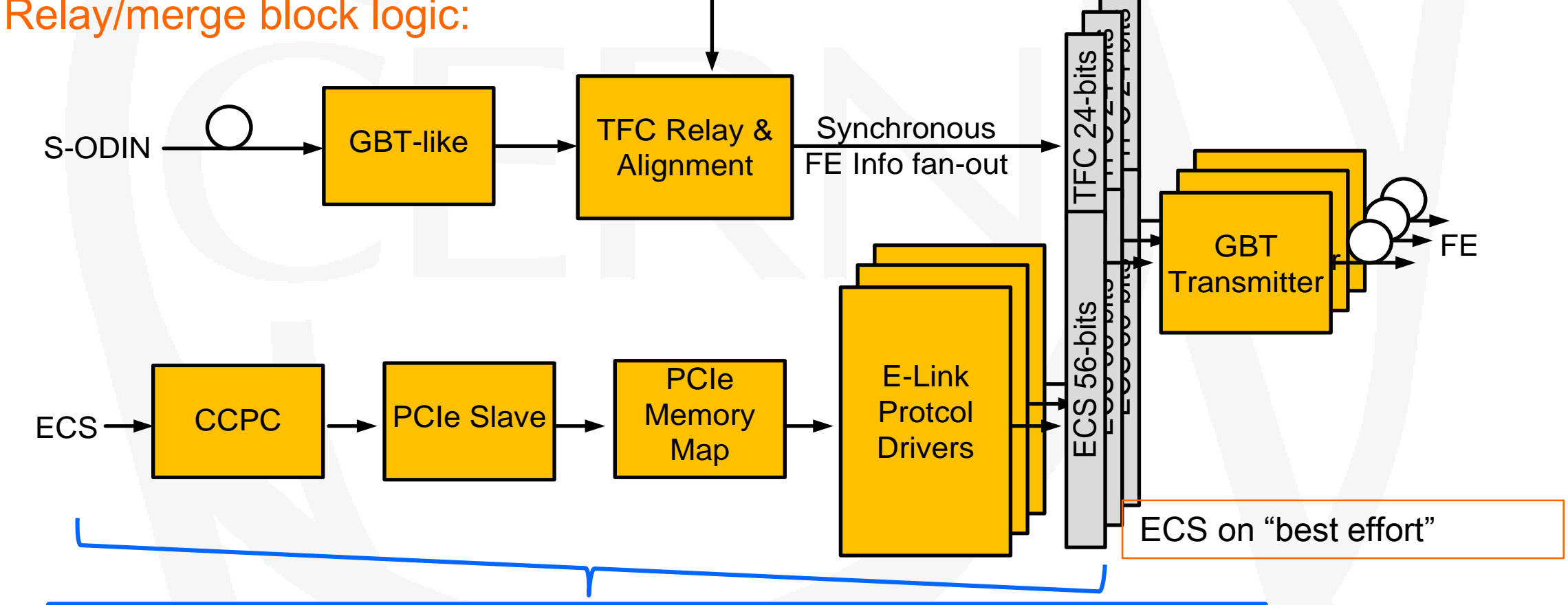

Generic approach:

$\rightarrow$ control FE from the uplink

$\rightarrow$ addressing map and GBT-busses protocol drivers

$\checkmark$ No need of special protocol, simply address the right chip with the right bus using the CERN GBT generic protocol

$\checkmark$ Same for every sub-detecto $r \rightarrow$ needs simply configuration 


\section{IHCh}

\section{Running a «hybrid» system}

Suggested the idea of an hybrid system:

reminder: some first-level trigger electronies relying on TTC protocol

$\rightarrow$ part of the system runs with old Timing and Trigger system

$\rightarrow$ part of the system runs with the new architecture

\section{How?}

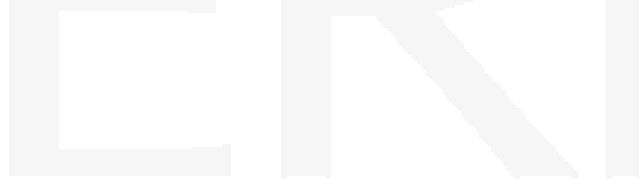

1. Need bidirectional connection between new Readout Supervisor (S-ODIN) and old Readout Supervisor (ODIN)

$\rightarrow$ use dedicated PICMG 3.8 compatible RTM board

2. In an early commissioning phase ODIN is the master, S-ODIN is the slave

$\rightarrow$ S-ODIN task would be to distribute new commands to new FE, to new TELL40s, and run processes in parallel

$\rightarrow$ ODIN tasks are the ones today + S-ODIN controls the upgraded part

$\checkmark$ In this configuration, upgraded slice will run at $40 \mathrm{MHz}$, but positive triggers will come only at maximum $1.1 \mathrm{MHz} \ldots$

- Great testbench for development + tests

3. In the final system, S-ODIN is the master, ODIN is the slave

$\rightarrow$ ODIN task is only to interface the L0 electronics path to S-ODIN and to provide clock resets on old Timing and Trigger protocol 


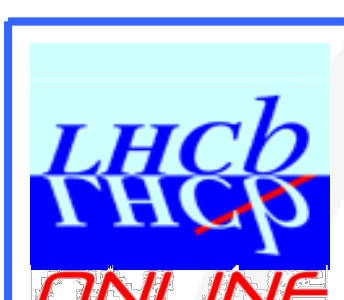

\section{Conclusions}

Outlined new Timing, Trigger and Readout Control system for the LHCb upgraded readout electronics:

$\checkmark$ Based on FPGAs and bidirectional optical links

$\checkmark$ Based on ATCA technologies and common readout electronics

$\checkmark$ To control the readotit by transmitting:

S. Synchronous commands

- Timing and clock with a controlled phase and fixed latency

- Trigger decisions

- Trigger throttle in case of back-pressure or readout load

- Events destination

$\checkmark$ To configure and monitor the FE electronics over the same link

- By using the CERNGBT protocol

- By having an addressing scheme and bus driving protocol directly into FPGAs

$\checkmark$ To allow running a hybrid system

- Old and new together!

$\checkmark$ Complete simulation and verification testbench under development

$\checkmark$ First version of the system to be ready by end of 2012 and first readout slice to be tested in 2013 


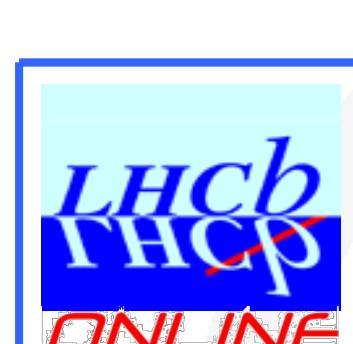

$m_{m}$

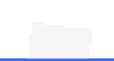

\section{Backup}

ONLINE 


\section{Readout Supervisor, specs}

$>$ Instantiate a set of TFC Master cores to guarantee partitioning control for subdetectors

$>$ TFC switches is a programmable patch fabric: a layer in FPGA

$\rightarrow$ no need of complex routing, no need of "discrete" electronics

$>$ Shared functionalities between instantiations (less logical elements)

$>$ More $\mathrm{I} / \mathrm{O}$ interfaces based on bidirectional transceivers

$\rightarrow$ depend on \#S-ROBs crates

$>$ No direct links to FE

$>$ Common server that talks directly to each instantiation:

$\rightarrow$ TCP/IP server in NIOS II

$>$ Flexibility to implement (and modify any protocol)

$\rightarrow$ GX transceiver as IP cores from Altera

$>$ Bunch structure (predicted/measured) rate control

$>$ State machines for sequencing resets and calibrations

$>$ Information exchange interface with LHC 


\section{TFC+ECSInterface, specs}

ONLINE

Board with FPGA entirely devoted to fan-out TFC information/fan-in throttle info

$>$ Controlled clock recovery

$>$ Shared network for Throttling (Intelligent) \& TFC distribution

$>$ All links bidirectional

$\rightarrow 1$ link to S-TFC Master, $2.4-3.0 \mathrm{~Gb} / \mathrm{s}$, optical

$\rightarrow 1$ link per S-ROB, 20 max per board (full crate)

$>$ Technology for S-ROBs links could be backplane (ex. xTCA) or copper HI-CAT

$>$ Protocol flexible: compatibility with flexibility of S-TFC Master

$\rightarrow$ We will provide the TFC transceiver block for S-ROBs' FPGA to bridge data to

FE through readout link S-FE $\rightarrow$ S-ROB

$>$ For stand-alone test benches, the Super-TFC Interface would do the work of a single TFC Master instantiation 


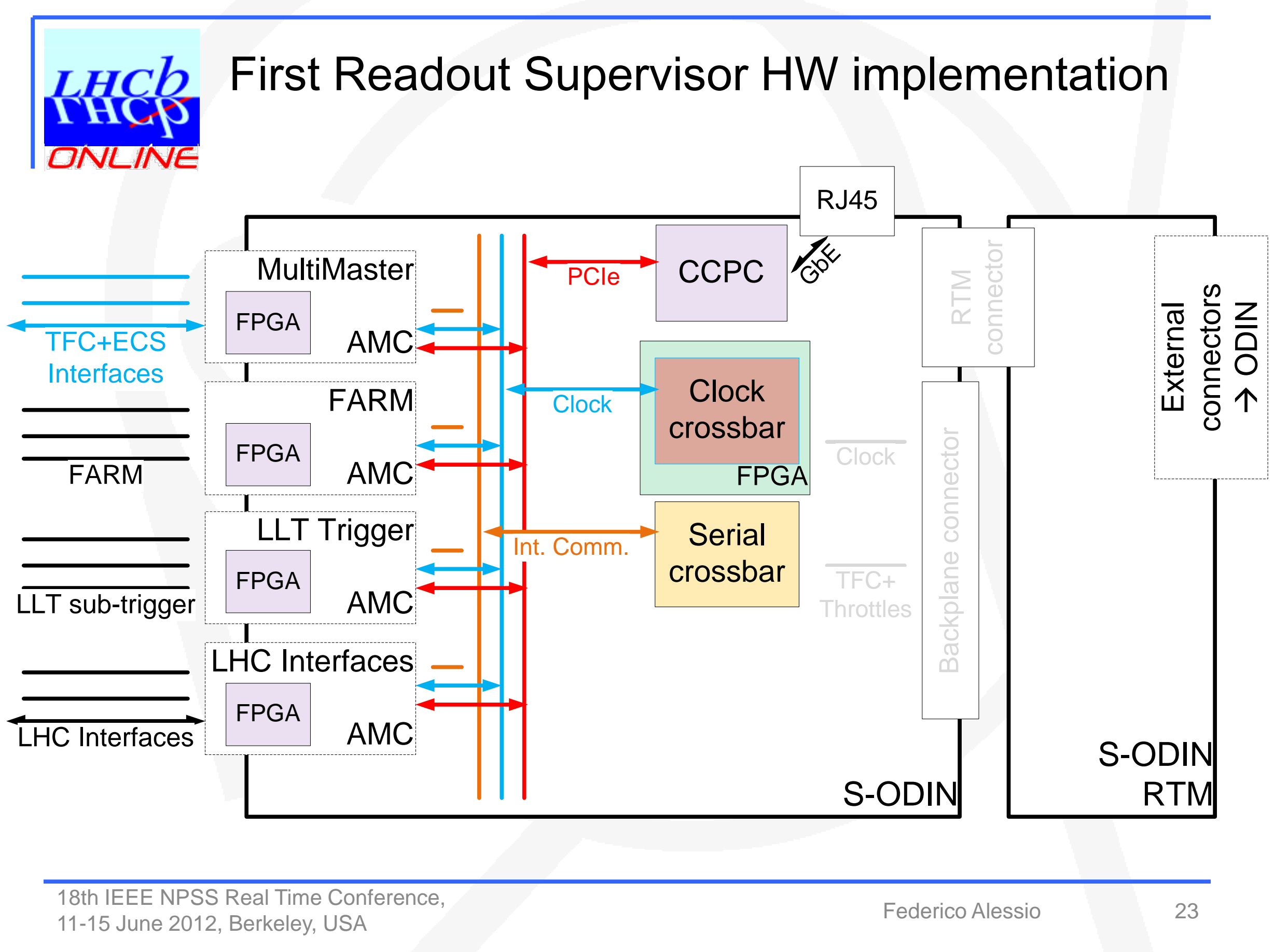




\section{THCP}

\section{LHCb First TFC+ECSInterface HW implementation}

ONLINE

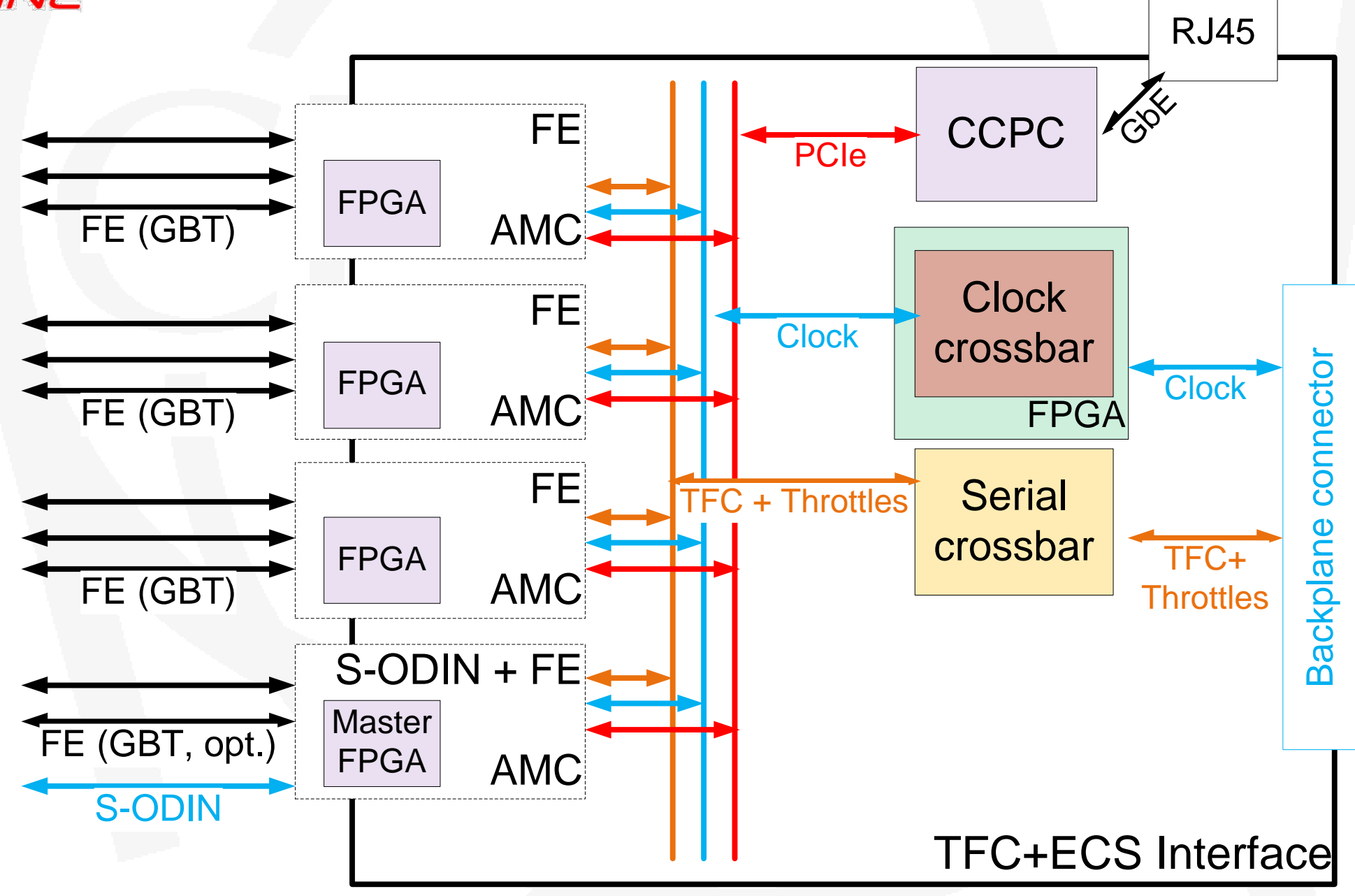




\section{$\mathrm{LHCb}$ Reaching the requirements: phase control}

1Mch

ONLINE
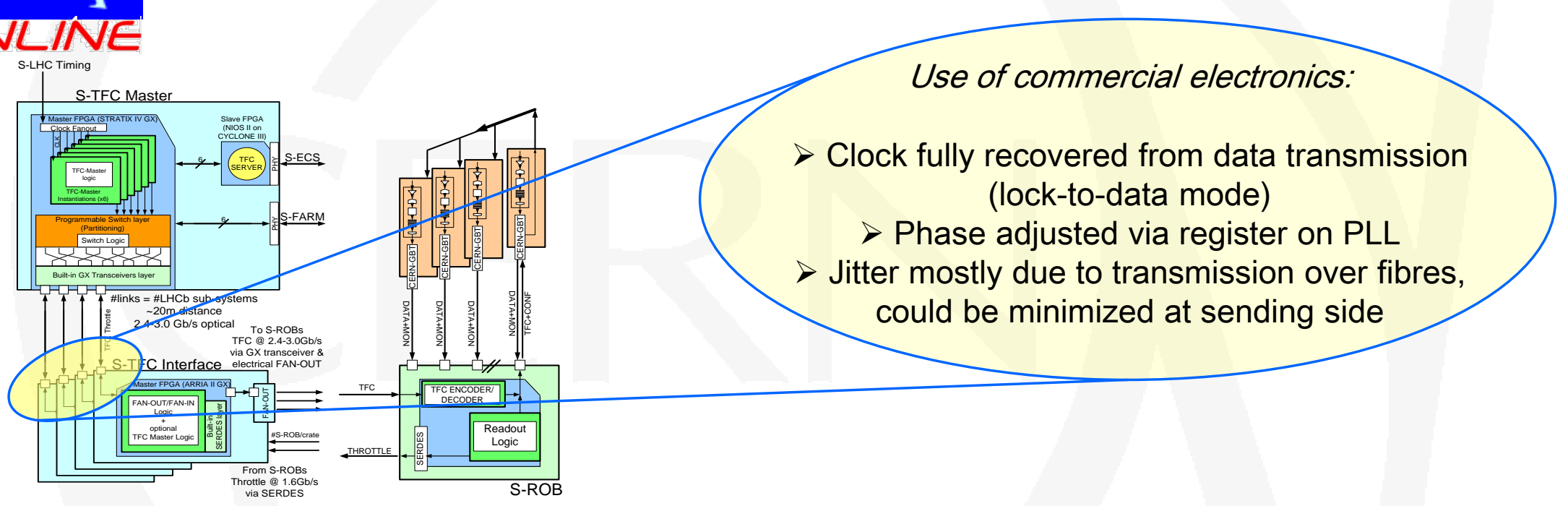

1. Use commercial or custom-made Word-Aligner output

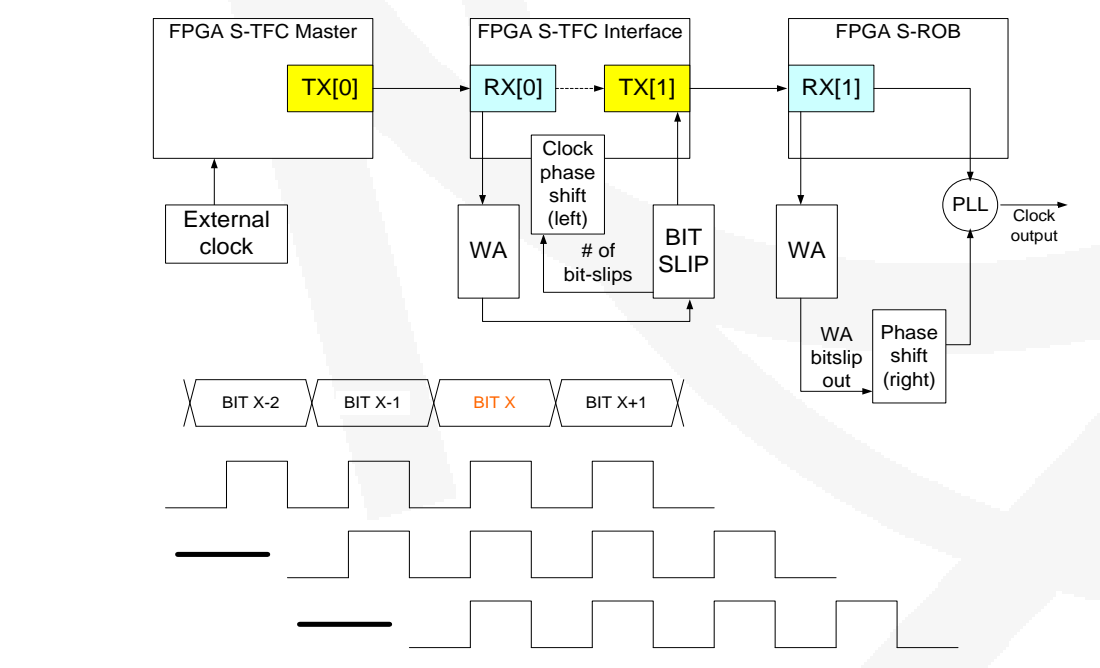

2. Scan the phase of clock within "eye diagram"

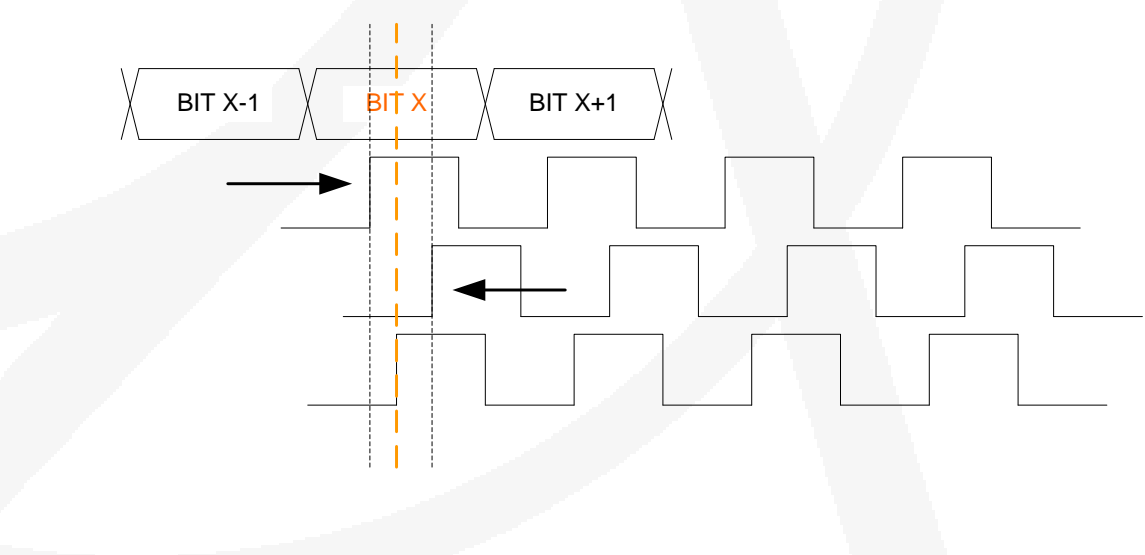




\section{IHCb}

ONLINE
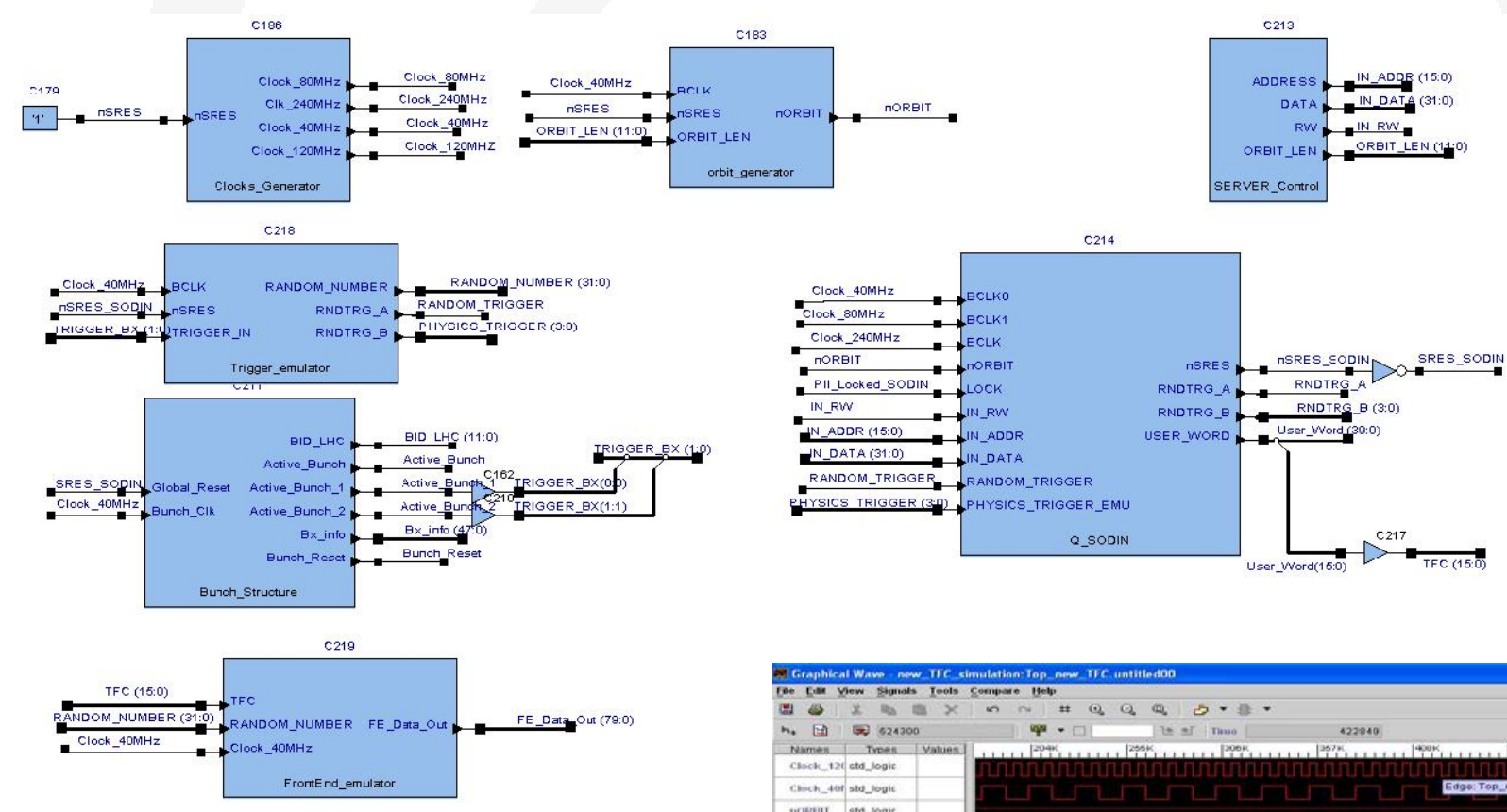

\section{Simulation}

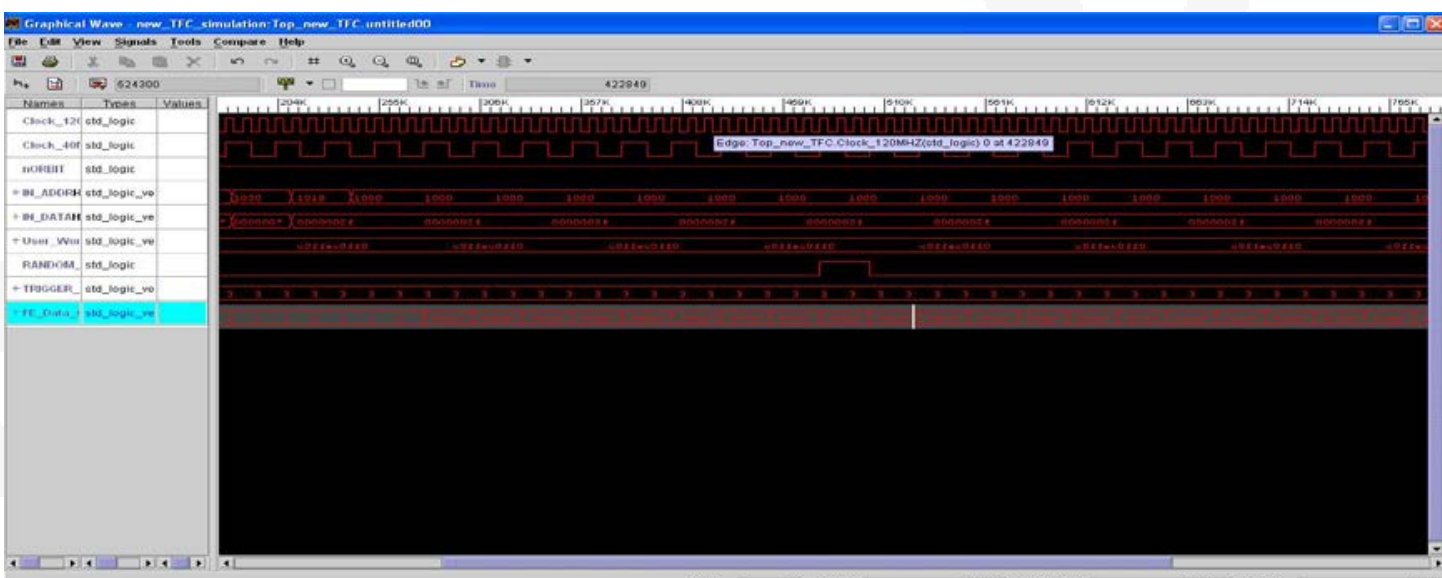

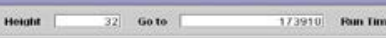

$\rightarrow$ Full simulation framework to study buffer occupancies, memories sizes, latency, configuration and logical blocks 


\section{LHCb Event Destination and Farm Load Control}

ONLINE

The current system operates in a powerful mixture of push and pull protocol controlled by ODIN:

$\checkmark$ Asynchronous pull mechanism

$\checkmark$ "Push" driven by trigger type and destination command

$\rightarrow 4$ years faultless operation

Similar proposal for upgrade

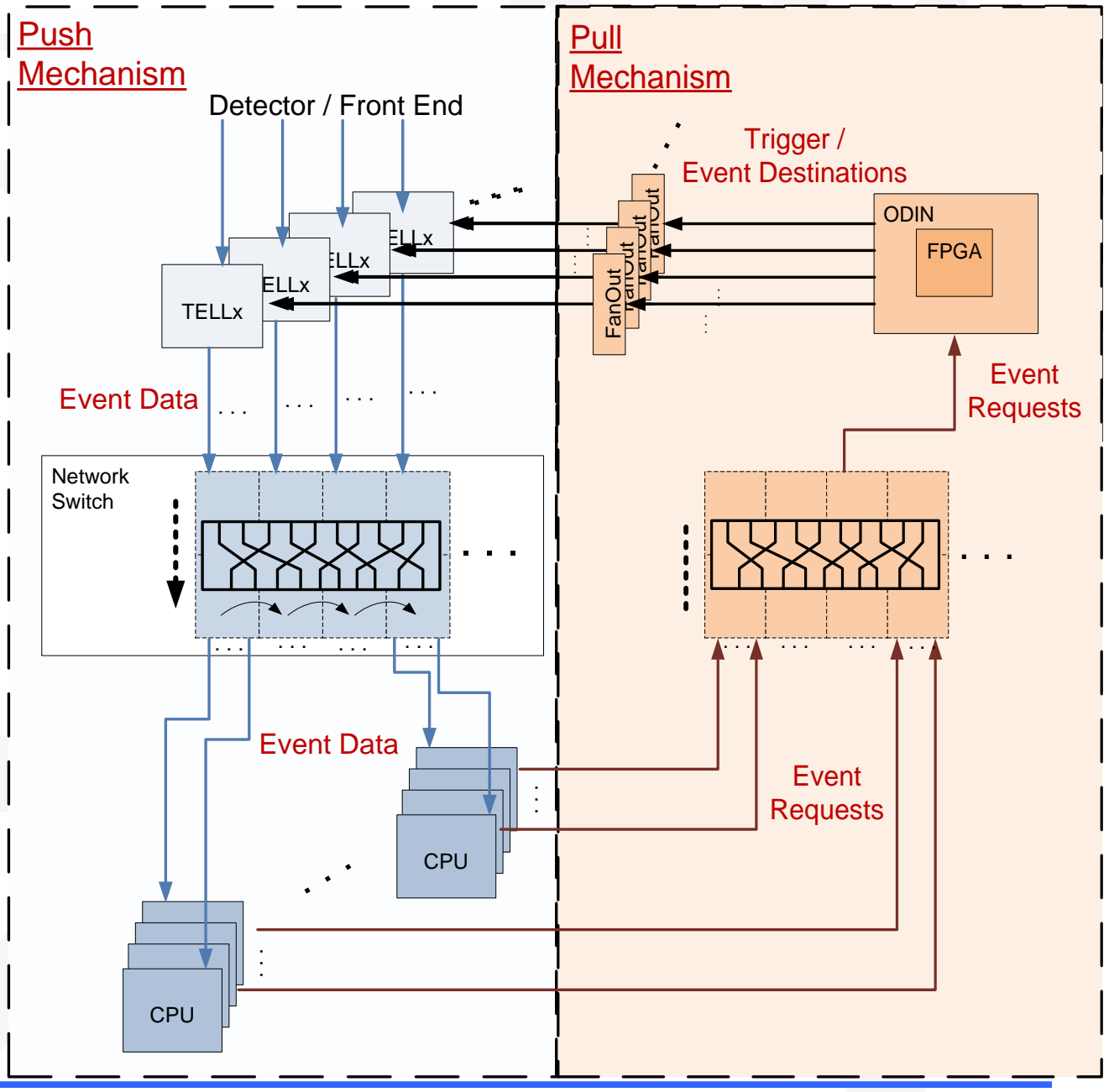




\section{LHCb Event Destination and Farm Load Control}

ONLINE

Central FPGA based implementation

- Extreme reliability, flexibility, speed, controllable latencies

$\rightarrow$ Central event packing control

- Different trigger types and destination types

- Variable MEP packing factor

$\rightarrow$ Dynamic rate regulation as function of farm rate capacity

- Accounts for statistical variations in processing time

$\rightarrow$ Dynamic handling of farm nodes in-flight

- Processing blockages, failures, interventions

- All impacts on rate capacity handled automatically

- As soon as nodes are recovered, included automatically in-flight by event request mechanism

$\rightarrow$ Minimal event loss and accurate dead-time counting

Contrary to conventional pull scheme, this is robust against event request packet losses 


\section{LHCb Event Destination and Farm Load Control}

aNlatNe

Buffer tequirement trivia

- Readout boards: 1.5 MEPs per link

- Network: Some naïve assumptions

- Rate: $30 \mathrm{MHz}$

- MEP packing factor $10 \rightarrow 3 \mathrm{MHz}$ MEPs and $3 \mathrm{MHz}$ MEP Requests

$\rightarrow$ Current ODIN can handle 1.8 MHz of MEP Requests (ODIN <-> FARM is $1 \mathrm{GbE} \ldots$..)

+ Event size $100 \mathrm{kB} \rightarrow 1 \mathrm{MB} / \mathrm{MEP}$

- Farm nodes $5000 \rightarrow 600 \mathrm{MEPs} /$ node/s $\rightarrow 1.7 \mathrm{~ms} / \mathrm{MEP}$

- Switch subunit sharing resources: 50 links / subunit $\rightarrow 100$ subunit

$\rightarrow 30 \mathrm{kHz}$ of MEPs per switch subunit

$\rightarrow$ Every $1.7 \mathrm{~ms}, 50 \mathrm{MEPs}$ to juggle with $\rightarrow$ < buffer> = O("50 MB")

$\rightarrow$ True need of memory depends on statistical variation of HLT processing time and "local farm derandomizer"

- Farm nodes: few MEPs in local derandomizing buffer

In our view, this looks like a straight-foryard implementation... 


\section{S-TFC Protocols}

> TFC control fully synchronous60bits@40MHz

$\rightarrow 2.4 \mathrm{~Gb} / \mathrm{s}$ (max 75 bits@ $40 \mathrm{MHz} \rightarrow 3.0 \mathrm{~Gb} / \mathrm{s})$

\begin{tabular}{|c|c|c|}
\hline BXID & TFC information & $\begin{array}{c}\text { ReedSolomon-FEC } \\
(12 \text { bits })\end{array}$ \\
\hline
\end{tabular}

$\checkmark$ Reed Solomon-encoding used on TFC links for maximum reliability $\rightarrow$ based on CERN-GBT

$\checkmark$ Asynchronous data

$\rightarrow$ TFC info carry Event ID

$\checkmark 24$ bits of TFC information relayed to FE electronics (see later) by TFC+ECSInterface

> Throttle protocol: each bit in Throttle is flagged by a Readout Board

\begin{tabular}{|c|c|c|c|}
\hline BXID & THROTTLE information \\
$(12$ bits $)$ & $(20$ bits $)$ & OTHERS & $\begin{array}{c}\text { ReedSolomon-FEC } \\
(16 \text { bits })\end{array}$ \\
\hline
\end{tabular}

$\checkmark \quad$ Must be synchronous (currently asynchronous)

$\rightarrow$ Protocol will require alignment between various input from Readout Boards

$\rightarrow$ Done in TFC+ECSInterface for each readout cluster 


\section{S-TFC protocol}

ONLINE

TFC Word to BE via TFC+ECSInterface:

$\neg 44$ bits (60 with Reed-Solomon encoder) @ 40 MHz = $1.76(2.4) \mathrm{Gb} / \mathrm{s}$

\begin{tabular}{|l|c|c|c|c|}
\hline Encoding & $43 . .32$ & $31 \ldots 16$ & 15 ..12 & 12.8 \\
\hline TFC Info & BID(11.0) & MEP Dest(15..0) & Trigger Type(3..0) & Calib Type(3..0) \\
\hline
\end{tabular}

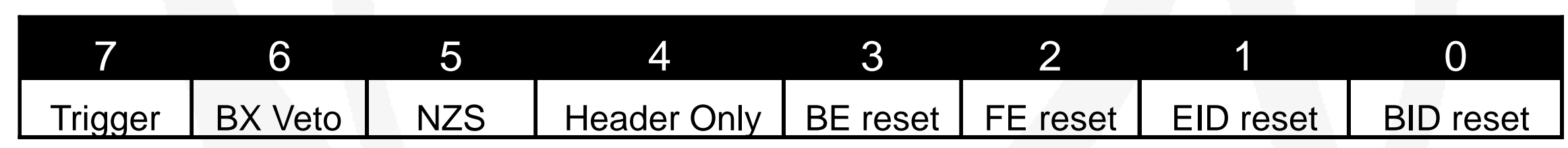

Constant latency after S-ODIN

$\checkmark$ THROTTLE Information from BE: 1 bit per board connected to TFC+ECSInterface. Full set of bits sent to S-ODIN by TFC+ECSInterface. 


\section{S-TFC protocol to FE!}

ONLINE

TFC Word to FE via TFC+ECSInterface:

$\neg 24$ bits in GBT frame @ $40 \mathrm{MHz}$

\begin{tabular}{|c|c|c|c|c|c|c|c|c|c|}
\hline Encoding & $23 . .12$ & $11 . .9$ & $8 . .5$ & 4 & 3 & 2 & 1 & 0 \\
\hline TFC Info & BID(11..0) & Reserve & Calib Type(3..0) & BX Veto & NZS & $\begin{array}{c}\text { Header } \\
\text { Only }\end{array}$ & $\begin{array}{c}\text { FE } \\
\text { reset }\end{array}$ & $\begin{array}{c}\text { BID } \\
\text { reset }\end{array}$ \\
\hline
\end{tabular}

$\checkmark$ 56bits leftover in GBT frame are dedicated to ECS configuration

$\checkmark$ uplink of GBT is dedicated to ECS monitoring of FE 


\section{LHCb S-TFC FE commands}

Crucial information: the decoding and sequencing (delays etc...) of these has to go in the FE design

ONLINE

"BXID" (+BXID Reset)

$\rightarrow$ Every TFC word carries a BXID for synchronicity of the system

"FE RESETS"

$\rightarrow$ Reset of Bunch Counter and Event Counter

"BX VETO"

$\rightarrow$ Based on filling scheme, processing of that particular event is rejected

$\checkmark$ Only header or basic bits sent from FE to TELL40s for that BXID

$\checkmark$ Allows "recuperating" clock cycles for processing "real" events

"HEADER QNLY"

$\rightarrow$ Idling the system: only header if this bit is set

$\rightarrow$ Multiple purposes (Resets, NZS scheme, etc...)

"CALIBRATION PULSES"

$\rightarrow$ Used to take data with special pulses (periodic, calibration)

$\checkmark$ Associated commands at fixed latency to FE

$\checkmark$ S-ODIN overrides LLT decision

"NZS MODE"

$\rightarrow$ Allows to read out all channels in FE (or all channels connected to a GBT)

$\checkmark$ Subsequent BXIDs are vetoed to allow packing of data into GBT frames

$\checkmark$ Only header or basic bits sent: use "Header Only" function 


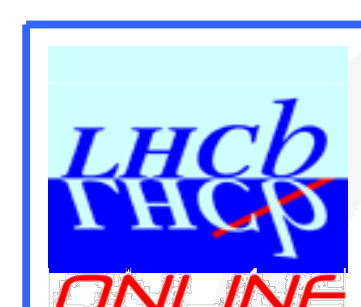

\section{«BX VETO»}

S-ODIN vetoes the readout of an event
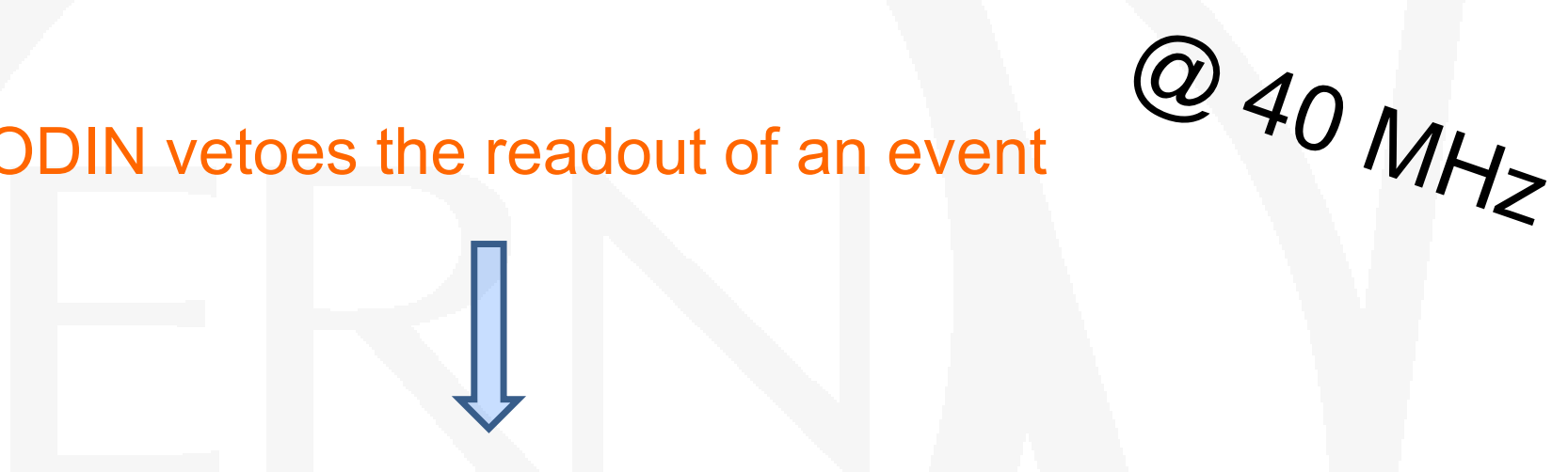

\section{Based on filling scheme}

$\rightarrow$ Used to control the rate of readout while $<30 \mathrm{MHz}$ $\rightarrow$ INDEPENDENT FROM LLT DECISION!

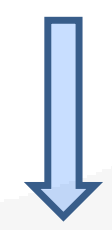

FE can use this info to recuperate time for processing events

$\rightarrow$ Only header for vetoed events

$\rightarrow$ Flexible packing of data into GBT frame 


\section{Sending a «LLTyes»}

ONLINE

(2)

S-ODIN receives decisions from LLT

Special triggers
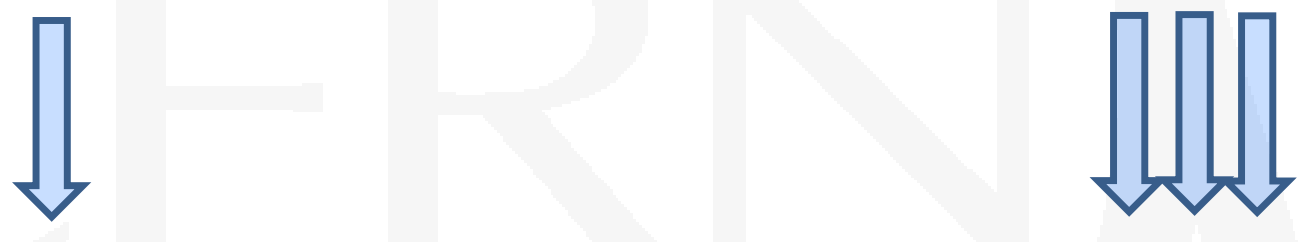

S-ODIN aligns and applies priority scheme on trigger types

Rate regulation (next slide)

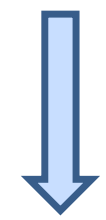

S-ODIN sends out a "LLTyes" to TELL40 at a fixed latency wrt BXID! 


\section{Rate regulation}

TELL40 raises the throttle bit

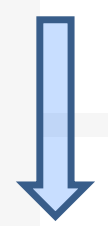

TFC Interfaces compiles a throttle word with BXID and sends it to S-ODIN
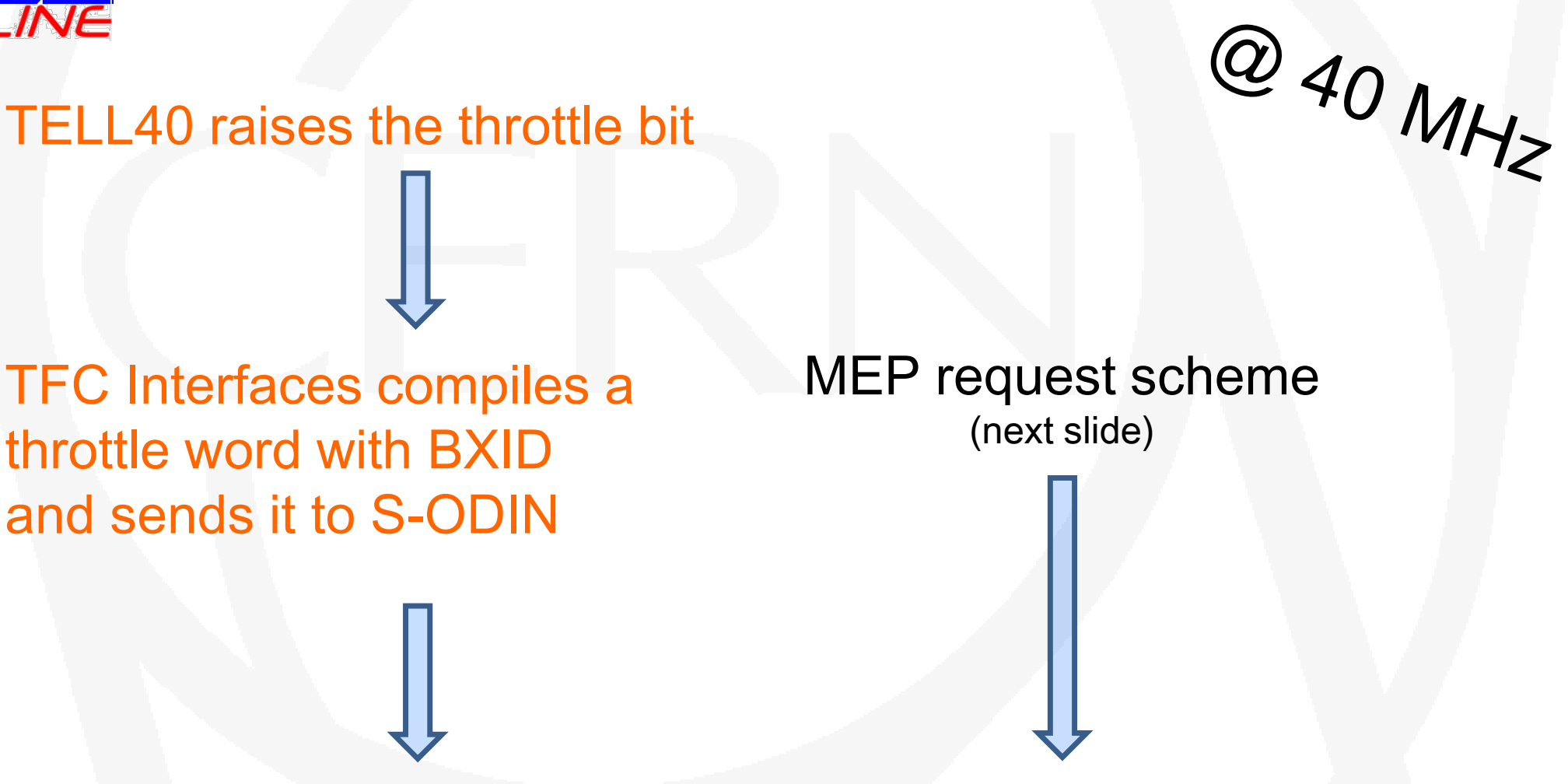

S-ODIN rejects event(s) until throttle is released $\rightarrow$ In practice: the subsequent "LLTyes"(s) become "LLTno"(s)! 